(c) 2016 IEEE. Personal use of this material is permitted. Permission from IEEE must be obtained for all other users, including reprinting/ republishing this material for advertising or promotional purposes, creating new collective works for resale or redistribution to servers or lists, or reuse of any copyrighted components of this work in other works. 


\title{
Building Detection Using Enhanced HOG-LBP features and Region Refinement Processes
}

\author{
Dimitrios Konstantinidis, Tania Stathaki, Member, IEEE, \\ Vasileios Argyriou, Member, IEEE, and Nikolaos Grammalidis, Member, IEEE
}

\begin{abstract}
Building detection from 2D high-resolution satellite images is a computer vision, photogrammetry and remote sensing task that has arisen in the last decades with the advances in sensors technology and can be utilised in several applications that require the creation of urban maps or the study of urban changes. However, the variety of irrelevant objects that appear in an urban environment and resemble buildings and the significant variations in the shape and generally the appearance of buildings render building detection a quite demanding task. As a result, automated methods that can robustly detect buildings in satellite images are necessary. To this end, we propose a building detection method that consists of two modules. The first module is a feature detector that extracts Histograms of Oriented Gradients (HOG) and Local Binary Patterns (LBP) from image regions. Using a novel approach, a Support Vector Machine (SVM) classifier is trained with the introduction of a special denoising distance measure for the computation of distances between HOG-LBP descriptors before their classification to the building or non-building class. The second module consists of a set of region refinement processes that employs the output of the HOG-LBP detector in the form of detected rectangular image regions. Image segmentation is performed and a novel building recognition methodology is proposed to accurately identify building regions, while simultaneously discard false detections of the first module of the proposed method. We demonstrate that the proposed methodology can robustly detect buildings from satellite images and outperforms state-of-the-art building detection methods.
\end{abstract}

D. Konstantinidis and T. Stathaki are with the Department of Electrical Engineering, Imperial College London, London, SW7

2AZ, United Kingdom e-mail: d.konstantinidis12@imperial.ac.uk, t.stathaki@imperial.ac.uk.

V. Argyriou is with the Department of Computer Sciences and Mathematics, Kingston University, Surrey, KT1 2EE, United Kingdom e-mail: Vasileios.Argyriou@kingston.ac.uk.

N. Grammalidis is with the Information and Technology Institute, CERTH, Thessaloniki, 57001, Greece e-mail: ngramm@iti.gr. 


\section{Index Terms}

Buildings, Satellite applications, Feature Extraction, Vegetation, Water, Image segmentation.

\section{INTRODUCTION}

Land cover classification is a widely-studied field since the appearance of the first satellite images. In the last two decades, the sensors attached to satellites have evolved in a way that nowadays allows the capture of high-resolution multi-spectral satellite images. This technological advance made the detection and classification of buildings and other man-made structures from satellite images possible. Building detection from satellite images can find usefulness in several remote-sensing applications, such as city planning, urban mapping and urban change detection. The knowledge of building locations can be proved valuable to municipalities in their efforts to assist, secure and protect their citizens, while illegal building construction activities can easily be detected and limited. Furthermore, urban expansion or decline can be studied and correlated to climatic changes and social, economic or natural factors and appropriate measures and precautions can be taken to ensure human prosperity.

Although building detection can be achieved manually by human experts, the tediousness of the process and the speed with which modern cities expand, make the development of automatic building detection algorithms imperative. Unfortunately, building detection from 2D multi-spectral images is a really challenging task. The fact that buildings appear in various sizes and shapes makes the development of a universal approach quite difficult. Furthermore, building rooftops in an urban environment may vary spectrally and there can even be spectral or texture variations in the same rooftop. The difficulty of building detection can further be magnified by the fact that relatively small buildings can be occluded by objects, such as trees and larger buildings. Moreover, weather conditions and sun location can severely affect the quality of a satellite image and therefore affect the building detection procedure. Although the existence of high-resolution multi-spectral images allows a lift in the burden of building detection by introducing accurate and more information-rich data, it also creates a difficulty in the processing of such enormous amount of data. Therefore, the development of robust, accurate and computationally fast building detection methods is of paramount importance.

Building detection from 2D images has been achieved using a variety of methods, where a building can be described either as a group of pixels sharing some common properties or as an 
object described by specific features or geometric properties. Pixel-based methods attempt to extract buildings by appropriately clustering image pixels into homogeneous regions. An overview of the most popular among these methods follows. Theng proposed an active contour algorithm to segment buildings from background. The initialization of the active contour algorithm was made using a circular cast algorithm [1]. A level-set segmentation approach to the building detection task, based on the notion that buildings can be described by certain characteristics (shape, colour, texture, etc) that allows the construction of a suitable energy function was suggested in [2]. Unfortunately, it is often hard or even impossible to construct an energy function that can characterize every building in an urban area, due to colour and shape variations buildings demonstrate. As in our approach, several other methodologies take advantage of the Normalised Difference Vegetation Index (NDVI) to separate man-made objects from vegetation. Singh et al. employed NDVI to remove vegetation and filtered the remaining image regions to keep only those with sizes in a range capable to represent building candidates [3]. Similar strategy was followed in [4] with the addition of an object-based classification procedure after the vegetation removal to differentiate blobs that belong to buildings from blobs that do not.

On the other hand, object-based methods identify features or extract shapes from an image that can characterize buildings. Sirmacek and Unsalan in [5] employed a building detection method based on the combination of Scale-invariant Feature Transform (SIFT) keypoints and graph theory. They used sub-graph matching to detect urban areas and graph cuts to identify separate buildings in an urban environment. In another work, the same authors developed a method to extract corners (Harris, Features from Accelerated Segment Test (FAST)), Gabor features and Gradient-Magnitude-based Support Regions (GMSR) from satellite and aerial images. They computed the kernel density estimation of these features and merged those using data and decision fusion schemes to locate building centers [6]. In several studies, lines proved to be significant features for the task of building detection. Lines can either be found by Hough Transform [7] or by detecting edges and forming edge chains. Edge chains were employed in [8] to identify lines, which were used at a later stage to form building candidates. Possibly missing lines were inferred and rectangles were formed. Another building detection method based on line grouping was attempted in [9], while in [10], the authors combined line grouping and corner labeling to form building hypothesis.

Parameterized shapes, namely templates, are used as an alternative way to solve the task 
of building detection. Vinson et al. in [11] used deformable templates of arbitrary scale and orientation to fit with the blobs extracted after applying a height threshold to a Digital Elevation Model. Karantzalos and Paragios in [12] combined a level-set segmentation approach driven by 2D shape priors to achieve building segmentation in urban areas. They demonstrated that the introduction of shape templates in a data-driven approach can improve the building detection results. Shadow detection has also been incorporated in several building detection methods, as a way to denote the existence of tall structures, which can be candidate buildings [4], [13]. However, shadow detection techniques can be significantly affected by the position of sun the time the image is captured.

The advances in the field of artificial intelligence have sparked the use of machine learning techniques to solve the problem of building detection. Super-pixels, the smallest clusters of pixels with similar multi-spectral information that can be formed, were employed in [14]. The authors used Conditional Random Fields to label super-pixels and form building candidates. Shackelford and Davis in [15] employed a pixel-based fuzzy classifier to label pixels in a multi-spectral image and a region merging segmentation procedure to split an image into meaningful disjoint sets of pixels. Afterwards, they used skeletonization and polygon approximation procedures to infer the boundaries of the identified buildings. Similarly, fuzzy logic inference with texture and line features was employed in [16] to detect buildings in an aerial image. To identify building regions, Senaras et al. in [17] extracted various spectral, texture and shape features, trained a base-layer fuzzy classifier for each feature and fused these classifiers' decisions by a meta-layer fuzzy classifier.

Chai et al. in [18] used a Markov Random Field (MRF) for low-level modeling of spectral data and Marked Point Processes for high-level modeling of buildings. They combined these two models and optimized the results using simulated annealing in order to segment buildings from the background. An MRF framework that exploits knowledge specific to the domain of buildings, such as shadow, rectangularity and vegetation was also employed in [19] to detect buildings. Finally, Femiani et al. in [20] took advantage of shadow information and vegetation constraints to drive a graphcut algorithm towards a successful building segmentation.

The methodology proposed in this paper is an object-based approach to the problem of building detection. It is a continuation and extension of our previous work [21], where HOG features [22] are extracted and trained using an SVM classifier. In this study, however, the HOG features 
are enhanced with the concatenation of LBP features [23], [24]. The combination of HOG and LBP features has been previously employed in various human detection tasks with great success [25], [26]. One of the main contributions of our work is the use of a novel special denoising measure that computes the distance between the HOG-LBP features in the SVM classifier. A cosine-based distance function was initially introduced by Fitch et al. in an attempt to robustly and accurately compute the translational displacements between video frames [27]. This distance function was found to allow for a suppression of the effects of noise and outliers and be more robust than its $l_{2}$-norm counterpart. Therefore, in this work, the SVM classifier is trained on the HOG-LBP descriptors using the above mentioned cosine-based distance function. Furthermore, we propose a novel and accurate region refinement procedure that receives the output of the HOG-LBP detector and outputs candidate building regions. To achieve this, image segmentation is performed using the Expectation-Maximization (EM) algorithm [28] and then image regions are selected as most probable to contain buildings. The selected regions are further processed and final building candidates are formed, while false alarms are rejected.

Our proposed strategy overcomes some of the inherent disadvantages of other techniques. Firstly, the parameters of the HOG-LBP algorithm are problem-specific and therefore, they can be set to achieve satisfactory results with images of different spatial or spectral resolution. Secondly, the HOG-LBP algorithm is robust to shape variations and can detect a variety of shapes, given that it is trained with a representative set of possible building shapes. Finally, we overcome one of the few inherent limitations of the HOG-LBP detector, which is its inability to accurately delineate building boundaries. To counter this, we propose a procedure that locates and extracts building regions from the detections of the HOG-LBP algorithm. As we will demonstrate, our proposed methodology performs better than other state-of-the-art algorithms that employ multiple features to solve the task of building detection.

In Section II, there is a detailed presentation of the proposed methodology and the two modules that it consists of: the HOG-LBP detector and the set of region refinement processes. The experimental evaluation of the algorithm and a comparison with other state-of-the-art methodologies is made in Section III. Finally, in Section IV, conclusions are drawn and suggestions for improvement of the proposed method are presented. 


\section{Proposed Methodology}

The proposed methodology can be split in two modules: the HOG-LBP detector and the region refinement procedure. The HOG-LBP detector is used both in the training and in the testing phase of the algorithm. HOG-LBP descriptors are extracted from the training images and employed for the training of an SVM model using the cosine-based distance function. Optimal Platt parameters [29] are also learned during the training phase to transform the SVM outputs to probabilities. In the testing phase, HOG-LBP descriptors are obtained from rectangular regions through dense scanning of an image and are classified in two classes, namely the building and non-building class. The region refinement procedure is employed only in the testing phase and is concerned with the segmentation of a tested image, the computation of the vegetation and water masks and the construction of the final building region candidates by selecting the most probable to correspond to a building image region for each positive HOG-LBP rectangular detection. The proposed methodology described briefly above is presented in Fig. 1, where the two modules along with their in-between interactions are illustrated. The training and testing phases of the algorithm are also depicted.

\section{A. HOG-LBP detector module}

In this section, the computation of the HOG-LBP descriptors is explained in detail. Moreover, the cosine-based distance function is analysed and arguments are presented in favor of its use as a kernel for the SVM classifier. Finally, Platt scaling is employed and a new threshold is introduced that improves the performance of the proposed HOG-LBP building detector.

1) HOG-LBP descriptor: The HOG descriptor is a successful and robust feature vector that was initially introduced as a means to detect pedestrians in an image [22]. Because of its superior discriminative power, it is selected as a suitable descriptor for building modelling and detection. A HOG descriptor is computed in an image region that is further divided into rectangular subregions, which are called cells. In each cell, a 1D histogram of the orientations of the intensity gradients present inside the cell is computed. The parameters that affect the computation of the HOG histograms can be optimally selected so that the developed HOG descriptor can differentiate image regions that contain buildings from those that do not.

The optimal parameters for the HOG descriptor in the task of building detection were selected based on our previous work [21]. However, for the sake of completeness, the most important 
parameters that affect the performance of the HOG building detector are also discussed here. Applying an unsharp masking before the HOG feature extraction is found to increase the classification accuracy of the HOG detector. The unsharped image, being the multiplied by a factor difference between the original and a blurred version of the image, is added to the original image in order to enhance details present in the image. Furthermore, histograms of gradients are computed for each channel of a multi-spectral satellite image separately and the resulted histograms are concatenated into a single histogram. A single rectangular kernel is proved to perform better than circular kernels or overlapping rectangular kernels. Finally, the extracted HOG descriptor are not normalised as the strength of the gradient magnitudes seems to be an important cue for the rejection of many of the false positives that the normalised HOG descriptor produces [21].

In this study, the HOG descriptor is enhanced with the introduction of LBP features [23]. LBP is a successful texture descriptor that is used in several computer vision applications to solve the task of object detection. It has been shown that when LBP features are used in conjunction with HOG features, their effect is complimentary, thus allowing for the development of a robust and accurate object recognition algorithm [25], [26]. Several LBP variants were implemented and tested in order to identify the LBP variant with the best discrimination ability for the building detection task when combined with the HOG features:

- Classical LBP: This is the initial LBP feature developed in [23], [24] and computed in a $3 \times 3$ pixel block of an image. The pixels in the block are thresholded by the center pixel's value, multiplied by powers of two and then summed to form the label of the center pixel. These pixel labels are employed in the computation of the classical LBP histograms.

- Uniform LBP: This LBP variant was formed based on the work in [30]. In their study, the probabilities of pattern occurrences were examined and it was deduced that some patterns appear more often than others in natural images [31]. The uniform LBP is computed exactly like the classical LBP with the difference being in the limited number of histogram bins.

- Center-Symmetric LBP (CS-LBP): The CS-LBP feature was developed in an attempt to reduce the length of the classical LBP descriptor. The CS-LBP descriptor takes into account the differences between pairs of pixels opposed symmetrically with respect to the center pixel. As a result, the CS-LBP descriptor is closely related to the gradient operator. In this way, the CS-LBP feature takes advantage of both the LBP descriptor and a gradient based 
feature [32].

- Improved D-LBP (ID-LBP): The ID-LBP was proposed in [33] as an improvement of the Directional LBP feature [34]. The ID-LBP descriptor is computed in a $3 \times 3$ pixel block and is based on the differences between the average of the pixels' values in the block and the pairs of the pixels opposed symmetrically with respect to the center pixel. The size of the ID-LBP descriptor is equal to the size of the CS-LBP descriptor.

LBP feature vectors are computed for each multi-spectral channel of a satellite image separately and concatenated in a large LBP descriptor. The computed HOG and LBP descriptors are then concatenated into a single HOG-LBP descriptor that is fed to an SVM classifier for training or classification. Experiments revealed that the LBP variant that achieves the best classification performance for the building detection task is the classical LBPs.

2) SVM classifier: One of the most significant contributions of this paper is the introduction of a cosine-based distance function as a distance measure to the SVM classifier. The cosinebased distance function was initially introduced by Fitch et al. to replace the $l_{2}$-norm function in the computation of displacement between video frames [27]. In this work, we propose the novel introduction of the cosine-based dissimilarity measure as a distance function for the training of the HOG-LBP descriptors by the SVM classifier. It is true that the $l_{2}$-norm dissimilarity measure has a significant flaw, namely, it is sensitive to outliers. The cosine-based dissimilarity measure is proved to be more robust to noise and outliers, thus being a more suitable distance function than the $l_{2}$-norm. Given that $\mathbf{x}_{\mathbf{i}}$ and $\mathbf{x}_{\mathbf{j}}$ are two feature vectors of length $L$, an ideal distance function can be approximated by a limited number $P$ of sinusoidal terms, giving rise to the cosine-based dissimilarity measure

$$
d\left(\mathbf{x}_{\mathbf{i}}, \mathbf{x}_{\mathbf{j}}\right) \approx \sum_{p=1}^{P} \sum_{l=1}^{L} b_{p}\left(1-\cos \left(a_{p} \pi\left(\mathbf{x}_{\mathbf{i}}(l)-\mathbf{x}_{\mathbf{j}}(l)\right)\right)\right)
$$

Because of the high computational cost of determining the coefficients $a_{p}$ and $b_{p}$ for more than one sinusoidal terms since several hundreds of classifiers need to be trained depending on required precision, we employ the single term $(P=1)$ distance function. Given also the constraint $\sum_{p=1}^{P} b_{p}=1$, we end up with a dissimilarity measure, where only the variable $a_{1}$ is unknown. This parameter, simply noted $\alpha$, represents the frequency of the cosine and it can be optimized to work best for the building detection task. 
The cosine-based dissimilarity function for various values of the parameter $\alpha$ and the $l_{2}$-norm distance function are presented and compared in Fig. 2. One may observe that small values of $\alpha$ make the cosine-based dissimilarity measure to resemble the $l_{2}$-norm, while increasing values of $\alpha$ allow the suppression of differences that could be attributed to noise and outliers. This holds because the cosine-based distance function for vector values in the range $[0,1]$ has an influence function (i.e. derivative) that is equivalent to Andrew's M-Estimate [27], [35]:

$$
\psi(r)= \begin{cases}\sin (\pi r) & \text { if }-1 \leq r \geq 1 \\ 0 & \text { otherwise }\end{cases}
$$

The Andrew's M-Estimate belongs to a category of outlier rejection techniques, called redescending m-estimators. The cosine-based distance function is considered outlier suppressor because it is not a monotonically increasing function as the difference between two vectors increases, but it redescends smoothly towards zero for large difference values. An example of outlier suppression is presented in Table I, where the histograms in the second and third rows are compared with the histogram of the first row using the $l_{2}$-norm and the cosine-based dissimilarity for various values of $\alpha$. The only difference between the first row and the second row histograms is located in the last bin, which could be attributed to noise, while the third row histogram is completely different than the first row histogram. As one may notice, the $l_{2}$-norm fails to capture the possible relations between the histograms, while this is not the case for the cosine-based dissimilarity measure with increased values of $\alpha$.

In this paper, the dissimilarity measure (1) is employed as a novel special denoising distance measure for the computation of distances between the HOG-LBP descriptors fed to the SVM classifier. To take advantage of the power of the cosine-based distance function, the HOGLBP descriptors should have values in the range $[0,1]$. To achieve this, we divide a HOG-LBP descriptor with the number of pixels used for its computation. This is possible because the gradient magnitudes have values in the range [0,1] for pixel values in the range $[0,1]$ and the LBP histograms are formed by counting the number of pixels belonging to the corresponding histogram bin. The optimal value of $\alpha$ is determined after exhaustive experimentation and is presented in the experimental section of this paper (Section III-D).

The cosine-based dissimilarity function for various values of the parameter $\alpha$ and the $l_{2}$-norm distance function are presented and compared in Fig. 2. One may observe that small values of $\alpha$ 
make the cosine-based dissimilarity measure to resemble the $l_{2}$-norm, while increasing values of $\alpha$ allow the suppression of differences that could be attributed to noise and outliers. An example of outlier suppression is presented in Table I, where the histograms in the second and third rows are compared with the histogram of the first row using the $l_{2}$-norm and the cosine-based dissimilarity for various values of $\alpha$. The only difference between the first row and the second row histograms is located in the last bin, which could be attributed to noise, while the third row histogram is completely different than the first row histogram. As one may notice, the $l_{2}$-norm fails to capture the possible relations between the histograms, while this is not the case for the cosine-based dissimilarity measure with increased values of $\alpha$.

In this paper, the dissimilarity measure (1) is employed as a novel special denoising distance measure for the computation of distances between the HOG-LBP descriptors fed to the SVM classifier. The optimal value of $\alpha$ is determined after exhaustive experimentation and is presented in the experimental section of this paper (Section III-D).

In the training phase, a set of annotated image regions that was manually and selectively cropped from the available original images are introduced to an SVM classifier with a radial basis kernel. However, instead of the $l_{2}$-norm, the cosine-based dissimilarity measure is employed as shown in (3). An SVM model is trained by determining the optimal parameters (cost of outliers $C$, kernel parameter $\gamma$ ) for which the cross-validation estimate of the test error is minimal. The trained SVM model is used for the classification in the testing phase.

$$
K\left(x_{i}, x_{j}\right)=e^{-\gamma \sum_{l=1}^{L}\left(1-\cos \left(\alpha \pi\left(x_{i}(l)-x_{j}(l)\right)\right)\right)}, \gamma>0
$$

Furthermore, Platt scaling is employed to transform the SVM scores to probabilities. Platt identified that class-conditional densities between the margins are exponential and two exponentials suggest the use of a parametric form of a sigmoid function [29]. Given that $x_{i}$ is the SVM output for a detection, the probability of this detection $p_{i}$ is defined as

$$
p_{i}=\frac{1}{1+e^{A x_{i}+B}}
$$

The constant terms $A$ and $B$ are determined iteratively by minimizing the negative loglikelihood of the training data 


$$
\min \left(-\sum_{i}\left(t_{i} \log \left(p_{i}\right)+\left(1-t_{i}\right) \log \left(1-p_{i}\right)\right)\right)
$$

where $t_{i}$ is equal to 0 for negative samples and 1 for positive samples. The purpose of the minimization is to fit the sigmoid function to the training data. The sigmoid parameters are determined using cross-validation on the training set. The idea behind the transformation of the SVM scores to probabilities is two-fold, namely to enhance the meaning of the output of the SVM classifier as instead of being the unbounded distance of a sample from the separating hyperplane, it now expresses the probability of a sample to belong to the positive ("building") class and also to use a new probabilistic threshold to differentiate between building and nonbuilding regions, instead of the zero threshold that the standard SVM classifier assumes. As it will be shown in Section III-B, Platt scaling increases the classification performance of the HOG-LBP building detector.

In the testing phase, each test image is split in overlapping regions of multiple sizes (scales) and a HOG-LBP descriptor is extracted for each image region. Afterwards, the HOG-LBP features are classified to the building and non-building classes using the SVM model that was obtained in the training phase of the methodology. The image regions that are classified by the SVM model in the "building" class consist the initial rectangular image regions where buildings possibly exist. The output of the HOG-LBP detector is sets of Cartesian coordinates and scales, which define rectangular regions, possible candidates for the presence of buildings and corresponding values that represent the confidence of the detections. Higher confidence is associated with higher possibility of a true detection. Overlapping detections are merged in single detections using the mean-shift algorithm, as proposed by Dalal [36]. The output of the mean-shift algorithm is used as input to the second module of the proposed methodology [see Fig. 7(b)].

\section{B. Region refinement module}

Initially, the image is split in homogeneous regions by employing an unsupervised pixel clustering technique. Vegetation and water masks are also extracted by employing a well-known vegetation and a novel water index respectively. By identifying vegetation and water, we can at a later stage discard building regions with a significant amount of pixels labeled as either vegetation or water. Afterwards, we take advantage of the output of the HOG-LBP detector to 
deduce the most probable to represent buildings image regions. These initial building candidates are further processed to form the final building candidates.

1) Vegetation mask: One of the most accurate and well-known methods to detect vegetation in multi-spectral images is the NDVI index [37]. The NDVI index has been successfully applied in numerous remote sensing applications as an indicator of vegetated areas. This index takes advantage of the near-infrared band that most satellites provide to identify where vegetation exists. The rationale behind the use of $N D V I$ is based on the fact that light is better absorbed from vegetation than man-made structures. Consequently, this index produces high positive values for vegetated regions and low positive or negative values for non-vegetated areas. $N D V I$ is computed using the near-infrared and red channels as shown below, where $\rho_{N I R}$ and $\rho_{R}$ are the near-infrared and red channels respectively.

$$
N D V I=\frac{\rho_{N I R}-\rho_{R}}{\rho_{N I R}+\rho_{R}}
$$

$N D V I$ is computed for each image pixel and then an optimal threshold is automatically determined using Otsu's method [38] in order to identify the pixels belonging to vegetation. The threshold is computed based on the minimization of the intra-class variance of the NDVI values between the pixels belonging to vegetation and those that do not. Pixels with values higher than the value of threshold are labeled as vegetation. As a result, a binary mask is formed, where vegetation pixels are highlighted. A morphological opening, followed by a morphological closing operation, is finally applied to remove small "holes" or "islands" produced in the binary vegetation mask [see Fig. 7(c)].

2) Water mask: Water extraction can be proved really useful for the task of building detection. An urban area may not depict a significant number of water bodies, nevertheless the water in urban environments is usually concentrated in swimming pools. Since swimming pools usually have rectangular shapes, a building detection method that relies on shape features can mistakenly consider swimming pools as candidate buildings. As a result, the detection of water bodies and the removal of swimming pools can lead to false alarm reduction of a building detection methodology. A commonly applied index that can differentiate the water class from other classes is the Normalized Difference Water Index ( $N D W I)$ [39]. The $N D W I$ index is computed using the green and near-infrared channels as shown below, where $\rho_{G}$ and $\rho_{N I R}$ are the green and 
near-infrared channels respectively.

$$
N D W I=\frac{\rho_{G}-\rho_{N I R}}{\rho_{G}+\rho_{N I R}}
$$

A low positive threshold, such as 0.3 , can be set and pixels with $N D W I$ values over that threshold can be labeled as water [39]. However, we noticed that a single threshold cannot work in certain images of our dataset as there are pixels that do not belong to water pools but are mistakenly recognized as such. To counter this, we propose a novel method to identify water in multi-spectral images. Our proposed method is based on the observation that water bodies usually show much higher values for the green and blue channels, rather than the red and nearinfrared channels. Given the red channel $\rho_{R}$, the green channel $\rho_{G}$, the blue channel $\rho_{B}$ and the near-infrared channel $\rho_{N I R}$, the proposed water mask is defined using the following set of binary operations

$$
\begin{aligned}
\text { WaterMask }= & \left(\rho_{G}>2 \rho_{R}\right) A N D\left(\rho_{G}>2 \rho_{N I R}\right) A N D \\
& \left(\rho_{B}>2 \rho_{R}\right) A N D\left(\rho_{B}>2 \rho_{N I R}\right)
\end{aligned}
$$

Although the proposed method of water extraction seems to miss a few pixels belonging to the water class, it rejects most of the false positives that the $N D W I$ index produces. Since our proposed methodology discards the regions that belong to the water class, a water detection method that presents a lower recall but a higher precision is preferable. The result of applying (8) is a binary mask, where water pixels are highlighted. A morphological opening, followed by a morphological closing operation, is finally applied to remove small "holes" or "islands" in the above mentioned binary water mask, in the same fashion as in the vegetation extraction procedure [see Fig. 7(c)]. An example from an image of our dataset, where the NDWI produces many false alarms that our proposed index avoids, is presented in Fig. 3.

3) Image segmentation: The proposed HOG-LBP detector cannot recognize building boundaries, but produces a set of candidate rectangular image regions where buildings are likely to exist. To make the transition from the extraction of these rectangular detections to the final delineation of buildings, an accurate image segmentation is necessary. The purpose of the proposed image segmentation procedure is the partitioning of the image to a set of connected-component regions, 
whose pixels share common multi-spectral properties. This procedure is extensively described below, while an outline of our image segmentation approach is presented in Fig. 4.

In our approach, we employ the EM algorithm [28], since it produces more homogeneous regions than the k-means algorithm. We rejected other image segmentation approaches due to their dependence on parameters different from the number of classes that we can optimally and automatically define as shown below. Since a fixed number of clusters cannot describe adequately the spectral variations in all satellite images of our dataset, we propose the use of gap statistic [40] to define in an unsupervised manner the optimal number of clusters for each tested image separately. The gap statistic can be considered as the statistical formalisation of the "elbow" heuristic technique, which considers as optimal the number of clusters that leads to an abrupt fall to the sum of point-to-cluster distances. Given that the image pixels $x_{i}, i=1 \ldots n$ are split into $k$ clusters $C_{r}, r=1 \ldots k$ and each cluster contains $n_{r}$ pixels, the within-dispersion measure (i.e. sum of point-to-point distances in a cluster over all clusters) is

$$
W_{k}=\sum_{r=1}^{k} \frac{1}{2 n_{r}} \sum_{i, i^{\prime} \in C_{r}}\left(x_{i}-x_{i^{\prime}}\right)^{2}
$$

The gap statistic is then computed using the within-dispersion measure (9) of the clustered image pixels and the observed within-dispersion measures $W_{k b}, b=1 \ldots B$ computed as in (9) from $B$ generated subsets of the image pixels, drawn uniformly from the initial set of image pixels.

$$
\operatorname{Gap}(k)=\frac{1}{B} \sum_{b=1}^{B} \log \left(W_{k b}\right)-\log \left(W_{k}\right)
$$

The use of the logarithm in the within-dispersion measures gives an interpretation as loglikelihood. The generation of the $B$ subsets of pixels leads to the formation of an expected reference distribution to which the initial set of pixels is compared. The larger the number of subsets $B$ is, the more accurate the reference distribution will be. Given the mean of the observed within-dispersion measures $m=(1 / B) \sum_{b} \log \left(W_{k b}\right)$ and their standard deviation $s d_{k}=$ $\left((1 / B) \sum_{b}\left(\log \left(W_{k b}\right)-m\right)^{2}\right)^{1 / 2}$, the optimal number of clusters $\hat{k}$ corresponds to the smallest value of $k$, for which it holds

$$
\operatorname{Gap}(k) \geq \operatorname{Gap}(k+1)-s d_{k} \sqrt{(1+1 / B)}
$$


As a result, the optimal number of clusters is equal to the smallest value of $k$, for which $\log \left(W_{k}\right)$ drops the farthest below the expected reference distribution [40]. The optimal number of clusters $\hat{k}$ is determined by employing the gap statistic on the output of the k-means algorithm for increasing values of $k$. The k-means algorithm is computationally faster than its EM counterpart especially for large values of $k$, thus it is our selected clustering approach at this stage. Afterwards, we use $\hat{k}$ to train $\hat{k}$ Gaussian models for the EM algorithm, which produces the final image segmentation. Each pixel is labeled based on their colour values, where instead of the Red-Green-Blue (RGB) colour space, the Hue-Saturation-Value (HSV) colour space is employed for two main reasons; unlike the RGB colour space, the components of the HSV colour space are independent of each other and the Hue $(H)$ and Saturation $(S)$ channels are intensity and shadow invariant, thus the segmentation procedure based on HSV produces more homogeneous regions within the underlying object boundaries. The near-infrared channel $(N I R)$ is also employed, along with the three HSV colour channels and participates in the image segmentation procedure. Due to the fact that the $H$ channel is expressed in degrees and has values in the range $[0,360]$, the transformation (12) is proposed so that the new values are in the range [0,1], as is the case for the other three spectral channels $(S, V, N I R)$.

$$
H_{\text {new }}=0.5-0.5 \cos \left(\frac{\pi H}{180}\right)
$$

The set of regions that the EM algorithm outputs are further processed by applying a floodfill operation that connects neighbouring pixels with the same label and stops at edge pixels detected by the Canny algorithm. Such a procedure helps towards the accurate and robust identification of object boundaries, since objects are usually distinguished by their edges. Unfortunately, this procedure results in the over-segmentation of some image regions. To counter this, a region merging procedure is afterwards applied, where each region with size smaller than a threshold $T_{\text {small }}$ is merged with one of its neighbouring regions, with which it has the best colour similarity. The threshold $T_{\text {small }}$, which represents the area of the smallest region we wish to retain, should be set to a value quite smaller than the smallest building that should be detected in order to retrieve even non-homogeneous in colour buildings that were split in more than one regions. The purpose of $T_{\text {small }}$ is to get rid of the really small regions that increase the complexity of the problem, while being insignificant for the building detection task. The remaining image regions 
make up the pool of the candidate building regions that our proposed novel building region detector will select [see Fig. 7(d)].

4) Building region detector: The purpose of the proposed novel building region detector is the selection of the image regions that have the highest likelihood to correspond to buildings based on the output of the HOG-LBP detector, their colour difference to their neighbouring regions and their rectangularity. The proposed building region detector aims at achieving a transition from the detections obtained using the HOG-LBP descriptors to the accurate pixel-based delineation of the buildings in a satellite image. A block diagram of the building region detector along with an example visualizing the employment of the proposed method on a HOG-LBP detection is presented in Fig. 5. In the next paragraphs, we present and thoroughly explain the steps that the proposed building region detector consists of.

The first step of the proposed methodology investigates the scoring of the image regions that are formed by the image segmentation procedure. Let assume that the proposed HOG-LBP detector is an ideal object detector, which possesses the following properties; (a) each detection corresponds to one and only object, (b) the detected area is of rectangular shape and its centroid is approximately the same as the centroid of the object (i.e. building) and finally (c) the size of the object is associated to the scale in which the detection is found. Taking these properties into consideration, we develop a novel approach for the scoring of image regions and the selection of the best image regions as building candidates. From each HOG-LBP detection, represented as a rectangular area $A_{D}$ with width and height $l$ (width equals height in our case) and centroid $O_{D}$, a single image region $A_{R}$, being the result of the image segmentation procedure described in Section II-B3, with mean multi-spectral colour vector $C_{R}$, centroid $O_{R}$ and bounded by a rotated rectangle $A_{B}$ is selected as initial building candidate region based on the addition of four terms. These terms are the amount of overlap between a region and the detection $M_{\text {over }}$, the region's rectangularity $M_{\text {rect }}$, the region's colour difference to its neighbours $M_{c d i f f}$ and the distance of the region's centroid to the centroid of the detection $M_{d i s t}$. The purpose of employing and summing all these terms is to aleviate problems where buildings may not have exact rectangular shapes or their contrast with the background is weak. Next, we present how the above mentioned terms are computed and why they are selected to form a scoring metric for the selection of an image region as building candidate.

- Overlap with HOG-LBP detection $M_{\text {over }}$ : The overlap of an image region with a HOG- 
LBP detection is equal to the area intersection between the image region and the detection divided by the union of the areas of the image region and the detection. Thus, the overlap term $M_{\text {over }}$ is equal to $A_{R} \cap A_{D} / A_{R} \cup A_{D}$. The use of this term is based on the notion that an ideal HOG-LBP detector should produce a detection large enough to cover the area of a true building and small enough to include minimum background. As a result, ground areas or roads, whose sizes can be large with respect to the sizes of the corresponding HOG-LBP detections are devalued.

- Rectangularity $M_{\text {rect }}$ : The term rectangularity is defined as the ratio of the area of an image region divided by the area of a rotated rectangle that bounds the image region. Consequently, the rectangularity term $M_{\text {rect }}$ can be computed by the ratio $\left|A_{R}\right| /\left|A_{B}\right|$. High values of rectangularity mean that the shape of an image region resembles a rectangle. Since the rectangle is the most common shape of buildings, this measure is an important term to differentiate between true buildings and false alarms.

- Colour difference $M_{\text {cdiff }}$ : The colour difference refers to the multi-spectral difference between the mean colour of an image region $C_{R}$ and the average weighted by size colour $C_{N}$ of its neighbouring image regions, which are defined by the region adjacency graph computed during the image segmentation procedure. Hence, the colour difference term $M_{\text {cdiff }}$ is defined as $\sqrt{\left\|C_{R}-C_{N}\right\|^{2}} / N$, where $N$ is the dimensionality of the colour vectors $C_{R}$ and $C_{N}$. The largest the colour difference is, the more likely it is that an image region distinguishes from its surroundings and it may represent a true building.

- Distance from detection's centroid $M_{\text {dist }}$ : This term is based on a property of an ideal object detector stating that the detections should be centered on the objects. As a result, the distance term $M_{\text {dist }}$ is equal to $e^{-2 \sqrt{\left\|O_{R}-O_{D}\right\|^{2}} / l}$. This means that the distance between the centroid of an image region that ideally represents a true building and the centroid of a detection should be as small as possible.

The image regions, whose centroids are located inside each rectangular HOG-LBP detection are considered. Afterwards, scores are computed for these regions based on the weighted sum of the terms defined above, using (13). The image region with the highest score is selected as initial building candidate. The weight vector $\mathbf{w}=\left[w_{1}, w_{2}, w_{3}, w_{4}\right]$ can be optimised by employing a grid search so that the contribution of the most reliable to describe buildings terms is boosted. 
The weight vector is computed using a validation set as it will be better clarified in Section III-D.

$$
\begin{aligned}
\text { score } & =\mathbf{w}\left[\begin{array}{c}
M_{\text {over }} \\
M_{\text {rect }} \\
M_{\text {cdiff }} \\
M_{\text {dist }}
\end{array}\right]=w_{1} \frac{A_{R} \cap A_{D}}{A_{R} \cup A_{D}}+w_{2} \frac{\left|A_{R}\right|}{\left|A_{B}\right|} \\
& +w_{3} \frac{\sqrt{\left\|C_{R}-C_{N}\right\|^{2}}}{N}+w_{4} e^{-\frac{2 \sqrt{\left\|O_{R}-O_{D}\right\|^{2}}}{l}}
\end{aligned}
$$

The next step of the proposed methodology concerns the processing of the initial building candidates [see Fig. 7(e)] to form complete building candidates. This processing step is essential so that non-homogeneous rooftops that have been split during segmentation in more than one homogeneous regions, are merged in a single building candidate. The construction of complete building candidates is based on a novel iterative procedure that is applied to each initial building candidate separately. However, before the iterative procedure initiates, each initial building candidate is checked in order to find whether a significant percentage of its pixels (more than a threshold $T_{\text {over }}$ ) belong to either vegetation or water by employing the previously computed vegetation and water masks. In such case, the initial building candidate is discarded as false alarm without further processing. Consequently, larger values of the threshold $T_{\text {over }}$ decrease the tolerance of the algorithm to accept as building candidates regions with significant amount of pixels classified as vegetation or water. Otherwise, the iterative procedure is executed during which, the regions neighbouring the building candidate that do not belong to either vegetation or water are searched and the one with the closest colour similarity with the building candidate is selected. If the merging of the two regions forms a region that achieves a larger measure of rectangularity than the initial region, then the region merging is performed and the merged region becomes the new initial building candidate for the next iteration of the algorithm. Otherwise, the algorithm terminates and the region $A_{R}$ that has been formed so far is checked under two conditions shown in (14) before it is accepted as a complete building candidate.

$$
\begin{aligned}
& \left|A_{R}\right| \geq T_{\text {small } \text { scale }_{0}} \\
& \left|A_{R}\right| \leq \frac{\left|A_{w}\right|}{F_{\text {dev }}} \text { scale }_{0}
\end{aligned}
$$


The use of these conditions is based on the fact that the HOG-LBP detector does not only provide information about the location of a building, but also about the size of the building. This information is stored at the scales of the detections and is employed in order to discard candidate buildings that are less likely to correspond to true buildings. Given the scale $s a l e_{0}$ of the largest HOG-LBP detection that encloses region $A_{R}$ and the initial searching window $A_{w}$, condition (14a) states that the area of the region $A_{R}$ should be larger or equal to the size of the smallest region that is retained from the image segmentation procedure (i.e. $T_{\text {small }}$ ) multiplied by scale $_{0}$. Condition (14b) states that the size of the region $A_{R}$ should be smaller or equal to the size of the largest rectangular HOG-LBP detection that encloses the region, divided by a factor of deviation $F_{d e v}$. The threshold $F_{d e v}$ has values in the range $[0,1]$ and controls the size of the accepted building candidates with respect to the size of a HOG-LBP detection, hence a large threshold limits the range of building sizes the algorithm accepts. The final output of the initial building candidate processing is a set of complete building candidates and their corresponding measures of rectangularity that are employed in the final part of the methodology. An outline of the algorithm that forms the complete building candidates is presented in Fig. 6.

Finally, the complete building candidates are tested whether their rectangularity is over a userdefined threshold $T_{\text {rect }}$. Ideally, we would like to retain building candidates with clear rectangular shapes (i.e. high values of rectangularity) and hopefully, most falsely detected building candidates will be discarded due to their low measures of rectangularity. The building candidates with rectangularity higher than the threshold $T_{\text {rect }}$ form the final output of our proposed methodology [see Fig. 7(f)]. The thresholds $T_{\text {over }}, F_{\text {dev }}$ and $T_{\text {rect }}$ have been set using the validation dataset in order to enhance the performance of the algorithm as it will be shown in Section III-D.

\section{EXPERIMENTAL RESULTS}

Our proposed methodology was tested on the Athens dataset of satellite images that is introduced in Section III-A and results are presented and discussed. Initially, we introduce and compare the variants of our proposed building detection method. Afterwards, our proposed method is compared with state-of-the-art building detection approaches and object detection methods that were adjusted and trained for the problem of building detection. Finally, we analyze the sensitivity of the proposed method to changes in parameter configuration and we discuss the advantages and limitations of our approach in the building detection task. 


\section{A. Dataset and Metrics}

The Athens dataset consists of 29 multi-spectral orthorectified satellite images, depicting urban areas of Athens in Greece. Most of these satellite images (17 in number) were captured by the QuickBird satellite, have 4 multi-spectral channels (Red, Green, Blue and Near-infrared) and present a spatial resolution of $0.6 \mathrm{~m}$ per pixel, while 12 images were taken by the WorldView-2 satellite, have 8 multi-spectral channels (Red, Green, Blue, Yellow, Coastal, Red-Edge, NIR1, NIR2) and present a spatial resolution of $0.5 \mathrm{~m}$ per pixel. The images of the Athens dataset were split in a validation set that consists of 5 images (3 QuickBird and 2 WorldView-2 images) and a test set that consists of 24 images (14 QuickBird and 10 WorldView-2 images). The training set was the same as the one employed in [21], consisting of 700 positive and 1400 negative manually labeled image patches that were segmented from a QuickBird image, different from the images used in the validation and test set. To be able to apply our proposed methodology to both types of satellite images without modifications, we employ only the 4 common multi-spectral channels. The test set contains 5105 buildings, annotated by human experts on separate ground truth masks.

The evaluation of the methodologies on the dataset is performed both at object and pixel level. We employ the common measures of recall, precision and $F_{1}$-score to measure performance [41], where

$$
\begin{gathered}
\text { Recall }=\frac{T P}{T P+F N} \\
\text { Precision }=\frac{T P}{T P+F P} \\
F_{1}-\text { score }=\frac{2 * \text { Recall } * \text { Precision }}{\text { Recall }+ \text { Precision }}
\end{gathered}
$$

In (15), $T P$ stands for true positives, $F P$ stands for false positives and $F N$ stands for false negatives. In our building detection framework, the object-based results are computed by counting the number of correctly detected and falsely identified buildings (TP and FP respectively). For pixel-based results, $T P$ stands for the number of correctly identified building pixels, $F P$ stands for the number of falsely labeled building pixels and $F N$ stands for the number of missed building pixels. 


\section{B. Comparison of HOG-LBP detector variants}

In this section, we analyze and compare the HOG-LBP detector variants that we implemented and tested on the satellite images of the Athens dataset. Our methodology is implemented using $\mathrm{C}++$ and the OpenCV library, from which several built-in functions have been employed. To detect buildings of various sizes in an image, all tested methodologies employ searching windows of multiple sizes (scales) ranging from $20 \times 20$ to $200 \times 200$ pixels. These sizes are adequate for the detection of buildings with sizes in the interval 50-8000 $\mathrm{m}^{2}$, based on the resolution of satellite images. The displacement between two consecutive searching windows (stride) is equal to 5 pixels, while the ratio between two consecutive scales is selected to be equal to 1.1 , as in our previous work [21].

The first differentiation in our proposed methodology comes with the introduction of the cosine-based dissimilarity measure as it was defined in (1). The computation of the distance between the HOG-LBP descriptors using the $l_{2}$-norm gives rise to the classical HOG-LBP detector, denoted simply as HOG-LBP detector, while the computation of the distance between the HOG-LBP descriptors using the cosine-based dissimilarity measure gives rise to the enhanced HOG-LBP detector. The second and final differentiation of the HOG-LBP detectors concerns the use of the Platt scaling. Instead of the typical SVM detector, where the detections with score higher than the threshold of zero are accepted as positive detections, the use of the Platt scaling allows the transformation of the SVM score to a probability. As a result, a new threshold can be set to differentiate between positive and negative detections. The new threshold, which is equal to 0.5 , means that we accept only the detections that have at least $50 \%$ probability to belong to the building class and has the advantage that it does not have to be determined manually by experimentation on the dataset.

These two differentiations give rise to four HOG-LBP detector variants (M1-M4), which are

implemented and tested in the images of the Athens dataset. The performance of the methods at object and pixel level are summarized in Table II, while the average object-based recall, precision and $F_{1}$-score and their corresponding standard deviations in the test set are presented in Fig. 9. The standard deviations are employed as an indicator of the robustness of the tested methods in the images of the Athens dataset. Since these images vary significantly in illumination and spatial resolution ( 2 types of satellite are used), we believe it is important to compute such a 
value.

From the results on the Athens dataset, one can deduce that the enhanced HOG-LBP detector with the employment of Platt scaling performs better than the other proposed HOG-LBP detectors. More specifically, the cosine-based distance function boosts the performance of our proposed method by $8.1 \%$ with respect to the measure of $F_{1}$-score. An additional $4.5 \%$ increase in $F_{1}$-score is achieved by Platt scaling. Although a slight drop in recall is observed when the cosine-based distance function and Platt scaling are employed, there is a significant increase in precision that leads to an overall better performance of the Enhanced HOG-LBP detector with Platt scaling (M4). The standard deviation of the Enhanced HOG-LBP detector with Platt scaling is lower than the other HOG-LBP detector variants by at least $15 \%$, meaning that this method is more robust to intensity and spatial resolution variations than the other methods in the Athens dataset.

As far as pixel-based performance is concerned, the Enhanced HOG-LBP detector with Platt scaling achieves similar performance to the HOG-LBP detector with Platt scaling and better performance than the cases, where no Platt scaling is introduced. Consequently, the additions of the cosine-based distance function and Platt scaling are successful in their attempt to remove noise and scale the SVM outputs respectively in a way to reduce false alarms and thus increase precision in the building detection task. These conclusions are further backed up by the comparison of the HOG-LBP variants in Fig. 10. The M4 method manages to reject most of the false alarms present at road segments that the other HOG-LBP variants accept, while it also achieves more precise boundaries around buildings. Moreover, all HOG-LBP variants (M1-M4) are capable of identifying problematic cases of buildings with spectral variations in their rooftops [see white circles in Fig. 10] or buildings partially covered by vegetation [see orange circles in Fig. 10].

\section{Comparison with other techniques}

In this section, our proposed methodology is compared with other object and building detection methodologies. We describe the implemented and tested methods and evaluate their performance on the satellite images of our test set.

- Cascade classifier with HAAR features (M5): A cascade of boosted classifiers is a structure of several simple classifiers, whose votes are aggregated to increase the performance of a 
detector. Such a cascade classifier that employs HAAR features was initially proposed in [42] and was later improved in [43]. The cascade classifier was trained and used for the detection of buildings in the Athens dataset. All four types of boosting techniques were tested, namely the Discrete Adaboost, the Real Adaboost, the Logitboost and the Gentle Adaboost. Furthermore, the classifier was tested for increasing number of cascade stages in order to identify which parameter configuration achieves the best performance on the building detection task. The results are presented in Fig. 8(a), where one can deduce that the Discrete Adaboost classifier with 16 cascade stages achieves slightly better results than the Real Adaboost with 16 stages and much better results than other parameter configurations.

- Cascade classifier with LBP features (M6): This classifier employs the same idea as the previously presented cascade classifier, with the only difference residing in the use of LBP features instead of HAAR features. Similar to the HAAR cascade classifier, experiments were run for all types of boosting techniques and several cascade stages in order to identify which parameter configuration achieves the best performance on the building detection task. The results are presented in Fig. 8(b), where one can deduce that the Logitboost classifier with 17 cascade stages achieves slightly better results than the Gentle Adaboost with 17 stages and much better results than other parameter configurations.

- Fisherfaces (M7): Fisherfaces is a method proposed to replace the Eigenfaces method as an alternative solution to the face recognition task by [44]. Using Fisher's Discriminant Analysis, class-specific dimensionality reduction is achieved in an attempt to increase the discrimination between classes and thus, the performance of an object detector. Fisherfaces were adjusted and employed for the task of building detection in the Athens dataset.

- Sirmacek et al. feature fusion (M8) [6]: A building detection methodology by fusing various distinctive features is proposed in [6]. The authors employed Harris and FAST corner features, GMSR regions and Gabor-filtering local features to identify building centers. Unlike the other algorithms, this is the only method that cannot detect building candidate regions but only building centers. Therefore, the performance of this methodology can only be evaluated at the object level and not at the pixel level. The object-level evaluation is based on single-pixel overlap of a computed building center with the ground truth building area, since the method does not provide any knowledge of the scale of the building.

- Konstantinidis et al. HOG detector (M9) [21]: In our previous work, we employed the 
HOG detector and used all multi-spectral information of a satellite image, concatenating the HOG descriptors computed for each multi-spectral channel separately. Furthermore, an optimal parameter configuration for the task of building detection was determined based on the validation set.

- Chaudhuri et al. building detector (M10) [45]: A building detector based on Internal Gray Variance (IGV) and morphology operations is proposed in [45]. IGV features are extracted and the edge pixels that do not correspond to man-made objects are rejected. Then, shadow detection is employed to remove false alarms and the remaining edges form the candidate regions out of which, buildings are extracted [45]. We implemented their method with the exception that their proposed shadow detection technique was replaced by the algorithm of Liu et al. [46] for panchromatic images as their shadow detection technique was not clear enough to be implemented. We experimented with various parameter values to find out that the values proposed in their paper yield the best results in the Athens dataset.

The performance of the previously described methodologies (M5-M10) is evaluated at object and pixel level in the Athens dataset and the total measures of recall, precision and $F_{1}$-score are summarized in Table II. Furthermore, the average object-based recall, precision and $F_{1}$-score and their corresponding standard deviations are presented in Fig. 9.

Methods M5-M9 can be described as object-based methods since no building delineation is attempted. Therefore, the performance of these methods can be directly compared to the performance of our proposed enhanced HOG-LBP detector with Platt scaling (M4). The results at the object level show the superiority of our object-based building detector (M4) over the other methods. More specifically, our proposed method achieves an increase in $F_{1}$-score by $10.7 \%$ over the second best method, which is the cascade classifier with LBP features (M6) and by more than $23 \%$ over the third best method, being the Konstantinidis et al. building HOG detector (M9). Worthy of notice is the almost perfect (marginally less than 1) recall of the Fisherfaces method (M7). However, the low precision of method M7 leads to mediocre $F_{1}$-score and thus, mediocre performance in the Athens dataset. Our proposed algorithm (M4) achieves the lowest standard deviation in $F_{1}$-score with a value of 0.058 , followed by the methods M5,M6 and M9 that perform similarly with a slightly larger value of 0.067 . This means that the method M4 shows greater robustness than the other methods with respect to illumination and spatial resolution changes present in the images of the Athens dataset. 
A comparison of the pixel-based performance of the methods M4-M9 reveals that the cascade boosted classifier with HAAR features (M5) achieves the best precision and $F_{1}$-score. However, the measure of recall is much lower than that of other methodologies, making it an undesirable building detector in the Athens dataset. Konstantinidis et al. HOG detector (M9) achieves the second best precision and $F_{1}$-score, but its recall is not as high as the recall that our proposed HOG-LBP detector (M4) achieves. The reason behind the low pixel-based precision of the methodologies M4-M7 and M9 is attributed to the fact that these methods detect regions where buildings are likely to exist and they cannot recognize and extract building boundaries.

A study of the tested methods M5-M9 and their corresponding results in the building detection task reveal their weaknesses. HAAR features sum up intensities in rectangular regions and thus they are sensitive to illumination changes, affecting significantly the results of building detection in images being under various lightning conditions. Fisherfaces method relies on the assumption that the classes can be linearly separated in an image subspace. However, this cannot be easily guaranteed in several object detection tasks. Sirmacek et al. method (M8) relies on the fusion of corner and gradient features. The images of the Athens dataset suffer from noise and low contrast of buildings to background and therefore, this method fails to extract enough corners to successfully identify building centers. Finally, the cascade classifier with LBP features (M6) and the Konstantinidis et al. HOG detector (M9) are successful in discriminating buildings from background but their performance is still outmatched by the complementary use of both HOG and LBP features employed in the proposed building detector (M4).

Next, we compare our proposed methodology with the addition of the region refinement procedure (M11) with another pixel-based building detection method (M10). These two methods are capable of accurately identifying and extracting buildings from satellite images. The results presented in Table II and Fig. 9 demonstrate the superiority of our proposed building detector (M11) over the algorithm developed by Chaudhuri et al. (M10) both at object and pixel level. More specifically, our method improves the object-based and pixel-based $F_{1}$-score by almost $12.8 \%$ and $69 \%$ respectively in comparison with the performance of the methodology M10 in the Athens dataset. Our methodology is inferior only in the object-based precision, where the algorithm M10 achieves the highest object-based precision from all tested object and pixelbased methodologies in the Athens dataset. Comparing the standard deviations, we conclude that our proposed methodology (M11) achieves the lowest standard deviation in $F_{1}$-score with 
a value of 0.041 , meaning that it is more robust than any other tested method, including the method of Chaudhuri et al. (M10). The building detection results of our proposed building detection framework (M11) and the algorithm of Chaudhuri et al. (M10) in two Quickbird and one WorldView-2 satellite images of the Athens dataset are visualized in Fig. 12.

The inferior performance of the Chaudhuri et al. building detector (M10) in the Athens dataset can be attributed to three main reasons. Firstly, the methodology is strongly affected by the selection of a suitable building template that enhances the contrast between the buildings and other objects in a satellite image. We experimented with various templates to get optimal results, but a selection of a non-representative template can significantly affect the results. Secondly, noise and low contrast present in the Athens dataset can severely affect the extraction of building edges that play a key role to the performance of the building detector. Finally, in high-resolution satellite images, shadow is present not only near buildings but also near other objects, such as tall trees and fences. As a result, non-building edges can be wrongly assumed to belong to buildings, leading to a drop in the performance of the building detector.

Next, our proposed pixel-based building extractor (M11) is compared with our proposed objectbased building detector (M4). The results show that the pixel-based performance of the algorithm M11 is significantly better than the performance of the method M4, as several false detections are removed and building boundaries are correctly identified. Unfortunately, some buildings are lost in the process, leading to a drop in the pixel-based recall and the object-based performance of the algorithm M11 in the Athens dataset. This loss of buildings can be attributed to the fact that the quality of image segmentation can be severely degraded in cases of noise in the satellite image or buildings with similar colour information to the background. Finally, there are some buildings with irregular shapes that do not present high rectangularity values and therefore, they are discarded either during the formation of the initial building candidates or by the employment of the rectangularity threshold.

Finally, Fig. 10 compares the detections of the tested methodologies in a part of a satellite images where buildings with spectral variations in their rooftops (i.e. buildings inside the white circles) and buildings partially occluded by vegetation (i.e. buildings inside the orange circles) exist. Results reveal that the cascade classifiers (methods M5 and M6), similar to the HOGLBP variants have no problem detecting such "problematic" cases of buildings. Unfortunately, the cascade classifiers seem to miss a few "regular" buildings or inaccurately identify them 
(i.e. significant portion of a detection is covered by background). The Fisherfaces method (M7) also manages to identify "problematic" cases of buildings, however at the expence of producing several false alarms around them. Sirmacek's approach (M8), on the other hand, shows a problem in identifying one of the buildings with spectral variations in its rooftop, while it also fails to detect several "regular" buildings. Similarly, both Konstantinidis et al. HOG detector (M9) and Chaudhuri et al. method (M10) fail to identify a building that presents spectral variations, while both algorithms successfully identify buildings that are partially covered by vegetation. Finally, our proposed methodology (M11) identifies correctly all the buildings that present either spectral variations in their rooftops or are partially occluded by vegetation. This can be attributed to the fact that the method M4 correctly captures such "problematic" cases of buildings and the region refinement procedure does not reject them as false alarms.

\section{Sensitivity to parameters}

Initially, experiments were made in order to determine which LBP variant achieves the greatest improvement in the performance of the building detector when it is combined with the HOG descriptor. The results of the experimentation with the LBP variants are presented in Table III and reveal that the best performance of our proposed building detector is achieved with the classical LBP feature vectors, followed by the slightly worse performance of the uniform LBP feature vectors.

We set the number of generated data subsets $B$ that affect the accuracy of the computed gap statistic equal to 20. Experiments revealed that the proposed methodology is robust to changes to the number of generated data subsets $B$ since high values affect slightly the accuracy of the gap statistic, while increase significantly the computational time. Furthermore, the morphological operations applied during the formation of the final vegetation and water masks are performed using a square structuring element of size $5 \times 5$ in pixels. This size is large enough to suppress small details and holes in the masks and small enough to avoid significant misclassification errors. The parameters that affect the performance of the proposed building detector and should be properly defined are the frequency of the cosine-based dissimilarity measure $\alpha$, the factor of deviation $F_{d e v}$, the minimum area of accepted image regions in the image segmentation procedure $T_{\text {small }}$, the percentage of building candidate's pixels that should belong to either vegetation or water so that the building candidate is discarded $T_{\text {over }}$, the weight vector $\mathbf{w}$ and the rectangularity 
threshold $T_{\text {rect }}$. These parameters are defined by experimentation on the 5 satellite images of our validation set and chosen based on the optimization of the pixel-based performance of our proposed building detector (M11).

The minimum area of an image region $T_{\text {small }}$ should be set smaller than the size of the smallest building, in order to take into account non-homogeneous rooftops. Since we have already employed a HOG-LBP building detector, $T_{\text {small }}$ can be set to a value proportional to the size of the initial searching window of the HOG-LBP algorithm. The weight vector $\mathbf{w}$ is determined by a grid search for component values in the range [0,1] with a step of 0.05 . This is possible because the absolute value of the weighted sum given by (13) is irrelevant for the comparison between the image regions as we are only interested for which region this sum gets the highest score. Experiments show that the most significant scoring term is the colour difference of an image region to its neighbours, followed by the overlap of an image region and the corresponding detection of the HOG-LBP algorithm. Finally, the parameter $\alpha$ that determines the frequency of the cosine-based distance function varies in the range $[0,1.99]$ with steps of 0.01 . Table IV presents the optimal parameter values that achieve the results presented in Table II. Fig. 11 illustrates the effects of varying the parameter values on the performance of the proposed methodology on the validation set.

From Fig. 11, a few conclusions can be drawn. The selection of an appropriate value for the frequency of the cosine-based dissimilarity measure $\alpha$ is of paramount importance. From Figs. 11 (a) and (b), it can be noticed that the performance of the proposed methodology increases only for values of $\alpha$ smaller than 0.4 . The optimal value of 0.09 for the parameter $\alpha$ means that the amount of noise in the training set is not significant and slight variations in the computed HOGLBP descriptors are essential for the differentiation between positives and negatives. From Figs. 11 (c), (d) and (f), one can observe that our proposed methodology is quite robust in changes of these parameters' values. Most of the regions with low rectangularity scores have already been rejected during the previous steps of the methodology and increasing the rectangularity threshold does not lead to a significant increase in the accuracy of the algorithm. In cases where the rectangularity threshold is set to too high values, a drop in the pixel-based performance of the proposed method is observed. Finally, the size of the smallest accepted image region seems to affect significantly the performance of the algorithm, as shown in Fig. 11 (e). This can be attributed to the fact that our proposed method is quite sensitive to the quality of the image 
segmentation, requiring an as accurate as possible image segmentation.

\section{E. Limitations}

Although our proposed methodology shows superior performance over other object-based and pixel-based building detection methods, there are still some significant limitations. Firstly, the results are significantly affected by the output of the HOG-LBP algorithm, meaning that buildings lost by the HOG-LBP detector cannot be recovered in the next steps of the proposed method. Secondly, the quality of image segmentation plays a crucial role to the building detection task. Buildings that are not accurately segmented by our proposed image segmentation procedure may not be selected by the region refinement procedure, thus being discarded by our proposed building detector. Cases of falsely or partially extracted buildings can be enhanced by the presence of noise and low contrast of buildings from background in satellite images. Although more than one scoring terms are aggregated, as shown in (13), there can still be non-building regions that achieve higher score than the building regions.

Finally, our methodology may fail to reject false positives that describe small road segments or land patches (see blue areas in roads and land in Fig. 12 (d)). In such occasions, the extracted image regions can present high rectangularity and strong contrast with the background, thus mistakenly recognised as buildings. Such problems can however be alleviated if steps are taken in the direction of segmenting a satellite image accurately, since then roads and land fields will extend out of the HOG-LBP detections and they will be devalued by the scoring metric (13).

\section{CONCLUSIONS}

In this paper, we propose a novel building detection methodology from satellite images. Our method employs concatenated HOG and LBP features using a novel distance function in the training and testing of the SVM classifier. Furthermore, we adopt a novel strategy to define a robust threshold using Platt scaling to determine which detections are accepted as positives and which are discarded as false alarms. The new threshold gives a significant boost to the performance of the HOG-LBP detector. Finally, we propose a novel approach to accurately detect building boundaries. The region refinement procedure is based on the result of an image segmentation procedure, the use of the output of the HOG-LBP detector and the employment of novel scoring metrics to determine the regions that are more likely to correspond to buildings. 
Applying our proposed method to the Athens dataset of satellite images reveals the superiority of the HOG-LBP detector over other object and building detection methodologies. The region refinement procedure, although successful in its purpose to improve the pixel-based performance of our building detector, faces problems keeping the high recall of the HOG-LBP detector, as a few buildings are lost in the process. Likely most of these problems can be mitigated by an accurate image segmentation procedure.

\section{ACKNOWLEDGEMENTS}

We would like to thank Dr. Beril Sirmacek for providing her code for our evaluation results. This research has been co-financed by the European Union (European Social Fund-ESF) and Greek national funds through the Operational Program "Education and Lifelong Learning" of the National Strategic Reference Framework (NSRF)-Research Funding Program: THALIS-NTUAUrbanMonitor. 


\section{REFERENCES}

[1] L. Theng, "Automatic building extraction from satellite imagery," Engineering Letters, vol. 13, no. 3, Nov 2006.

[2] K. Karantzalos and D. Argialas, "A region-based level set segmentation for automatic detection of man-made objects from aerial and satellite images," Photogrammetric Engineering and Remote Sensing, vol. 75, no. 6, pp. 667-677, 2009.

[3] D. Singh, R. Maurya, A. Shukla, M. Sharma, and P. Gupta, "Building extraction from very high resolution multispectral images using NDVI based segmentation and morphological operators," in Students Conference on Engineering and Systems (SCES), March 2012, pp. 1-5.

[4] T. Hermosilla, L. Ruiz, J. Recio, and J. Estornell, "Evaluation of automatic building detection approaches combining high resolution images and LiDAR data," Remote Sensing, vol. 3, no. 6, pp. 1188-1210, 2011.

[5] B. Sirmacek and C. Unsalan, "Urban-area and building detection using SIFT keypoints and graph theory," IEEE Transactions on Geoscience and Remote Sensing, vol. 47, no. 4, pp. 1156-1167, April 2009.

[6] _ - "A Probabilistic Framework to Detect Buildings in Aerial and Satellite Images," IEEE Transactions on Geoscience and Remote Sensing, vol. 49, no. 1, pp. 211-221, Jan 2011.

[7] P. Hough, "Method and means for recognizing complex patterns," U.S. Patent 3,069,654, Dec 1962.

[8] T. Kim and J. Muller, "Development of a graph-based approach for building detection," Image and Vision Computing, vol. 17, no. 1, pp. 3-14, 1999.

[9] D. Haverkamp, “Automatic building extraction from IKONOS imagery," in Proceedings of ASPRS, 2004.

[10] D. Woo, Q. Nguyen, Q. Nguyen Tran, D. Park, and Y. Jung, "Building detection and reconstruction from aerial images," in ISPRS Congress, Beijing, 2008.

[11] S. Vinson, L. Cohen, and F. Perlant, "Extraction of rectangular buildings in aerial images," in Proceedings of Scandinavian Conference on Image Analysis (SCIA), 2001.

[12] K. Karantzalos and N. Paragios, "Recognition-driven two-dimensional competing priors toward automatic and accurate building detection," IEEE Transactions on Geoscience and Remote Sensing, vol. 47, no. 1, pp. 133-144, Jan 2009.

[13] O. Benarchid, N. Raissouni, S. Adib, A. Abbous, A. Azyat, N. Achhab, M. Lahraoua, and A. Chahboun, "Building extraction using object-based classification and shadow information in very high resolution multispectral images, a case study: Tetuan, Morocco," Canadian Journal on Image Processing and Computer Vision, vol. 4, no. 1, Jan 2013.

[14] S. Kluckner and H. Bischof, "Image-based building classification and 3d modeling with super-pixels," in Proceedings of International Society for Photogrammetry and Remote Sensing, Photogrammetric Computer Vision and Image Analysis, 2010 .

[15] A. Shackelford and C. Davis, "A combined fuzzy pixel-based and object-based approach for classification of high-resolution multispectral data over urban areas," IEEE Transactions on Geoscience and Remote Sensing, vol. 41, no. 10, pp. 2354-2363, Oct 2003.

[16] Z. Sheng-hua, H. Jian-jun, and X. Wei-xin, “A new method of building detection from a single aerial photograph,” in 9th International Conference on Signal Processing (ICSP), Oct 2008, pp. 1219-1222.

[17] C. Senaras, M. Ozay, and F. T. Y. Vural, "Building Detection With Decision Fusion," IEEE Journal of Selected Topics in Applied Earth Observations and Remote Sensing, vol. 6, no. 3, pp. 1295-1304, June 2013.

[18] D. Chai, W. Förstner, and M. Ying Yang, "Combine markov random fields and marked point processes to extract building from remotely sensed images," in ISPRS Annals of the Photogrammetry, Remote Sensing and Spatial Information Sciences, 2012, pp. 1219-1222. 
[19] Ö. Ö. Karadağ, C. Senaras, and F. T. Y. Vural, "Segmentation Fusion for Building Detection Using Domain-Specific Information," IEEE Journal of Selected Topics in Applied Earth Observations and Remote Sensing, vol. 8, no. 7, pp. 3305-3315, July 2015.

[20] J. Femiani, E. Li, A. Razdan, and P. Wonka, "Shadow-Based Rooftop Segmentation in Visible Band Images," IEEE Journal of Selected Topics in Applied Earth Observations and Remote Sensing, vol. 8, no. 5, pp. 2063-2077, May 2015.

[21] D. Konstantinidis, T. Stathaki, V. Argyriou, and N. Grammalidis, "A probabilistic feature fusion for building detection in satellite images," in Proceedings of the 10th International Conference on Computer Vision Theory and Applications, 2015, pp. 205-212.

[22] N. Dalal and B. Triggs, "Histograms of oriented gradients for human detection," in IEEE Conference on Computer Vision and Pattern Recognition (CVPR), vol. 1, June 2005, pp. 886-893.

[23] T. Ojala, M. Pietikainen, and D. Harwood, "Performance evaluation of texture measures with classification based on Kullback discrimination of distributions," in International Conference on Pattern Recognition, vol. 1, Oct 1994, pp. 582585.

[24] — - "A comparative study of texture measures with classification based on featured distributions," Pattern Recognition, vol. 29, no. 1, pp. 51-59, Jan 1996.

[25] X. Wang, T. Han, and S. Yan, “An HOG-LBP human detector with partial occlusion handling," in International Conference on Computer Vision, Sep 2009, pp. 32-39.

[26] J. Zhang, K. Huang, Y. Yu, and T. Tan, "Boosted local structured HOG-LBP for object localization," in International Conference on Computer Vision and Pattern Recognition (CVPR), June 2011, pp. 1393-1400.

[27] A. Fitch, A. Kadyrov, W. Christmas, and J. Kittler, "Fast Robust Correlation," IEEE Transactions on Image Processing, vol. 14, no. 8, pp. 1063-1073, 2005.

[28] A. Dempster, N. Laird, and D. Rubin, “Maximum Likelihood from Incomplete Data via the EM Algorithm,” Journal of the Royal Statistical Society, vol. Series B 39, no. 1, pp. 1-38, 1977.

[29] J. Platt, "Probabilistic Outputs for Support Vector Machines and Comparisons to Regularized Likelihood Methods," in Advances in Large Margin Classifiers. MIT Press, 1999, pp. 61-74.

[30] F. Bianconi and A. Fernndez, "On the Occurrence Probability of Local Binary Patterns: A Theoretical Study," Journal of Mathematical Imaging and Vision, vol. 40, no. 3, pp. 259-268, 2011.

[31] M. Pietikainen, A. Hadid, G. Zhao, and T. Ahonen, "Local Binary Patterns for Still Images," in Computer Vision Using Local Binary Patterns, ser. Computational Imaging and Vision. Springer London, 2011, vol. 40, pp. $13-47$.

[32] J. Xiao and G. Wu, “A Robust and Compact Descriptor Based on Center-Symmetric LBP," in International Conference on Image and Graphics (ICIG), Aug 2011, pp. 388-393.

[33] S. Junding, Z. Shisong, and W. Xiaosheng, "Image retrieval based on an improved CS-LBP descriptor," in IEEE International Conference on Information Management and Engineering (ICIME), April 2010, pp. 115-117.

[34] W. Xiaosheng and S. Junding, "An effective texture spectrum descriptor," in International Conference on Information Assurance and Security (IAS), vol. 2, Aug 2009, pp. 361-364.

[35] P. Huber, Robust Statistics, ser. Wiley Series in Probability and Statistics. New York: Wiley, 1981.

[36] N. Dalal, "Finding People in Images and Videos," Ph.D. dissertation, National Polytechnique de Grenoble, July 2006.

[37] J. Rouse, R. Haas, J. Scheel, and D. Deering, "Monitoring Vegetation Systems in the Great Plains with ERTS," in 3rd Earth Resource Technology Satellite (ERTS) Symposium, vol. 1, 1974, pp. 48-62. 


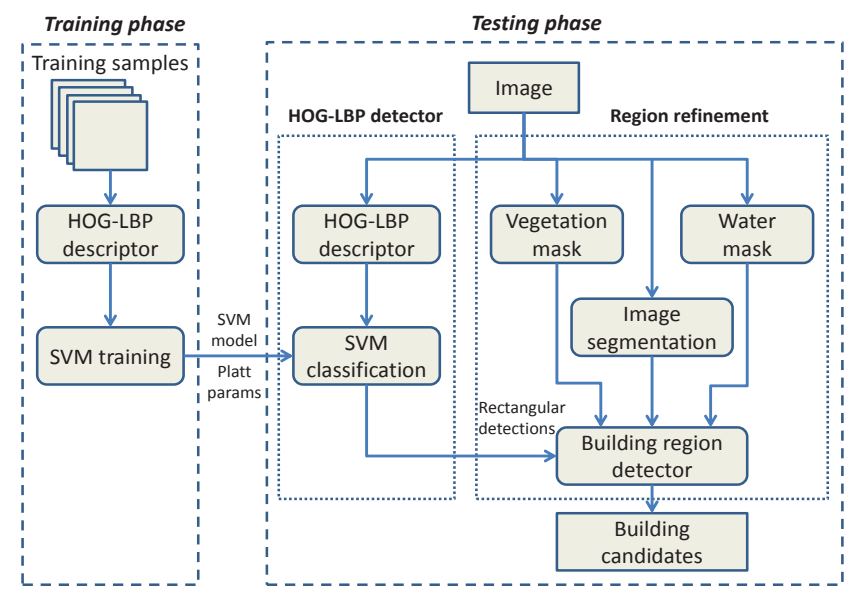

Fig. 1. Block diagram of our proposed methodology, along with the defined modules and their interactions.

[38] N. Otsu, "A Threshold Selection Method from Gray-Level Histograms," IEEE Transactions on Systems, Man and Cybernetics, vol. 9, no. 1, pp. 62-66, Jan 1979.

[39] S. McFeeters, "Using the Normalized Difference Water Index (NDWI) within a Geographic Information System to Detect Swimming Pools for Mosquito Abatement: A Practical Approach,” Remote Sensing, vol. 5, pp. 3544-3561, 2013.

[40] R. Tibshirani, G. Walther, and H. T., "Estimating the number of clusters in a data set via the gap statistic," Journal of the Royal Statistical Society: Series B (Statistical Methodology), vol. 63, pp. 411-423, 2001.

[41] D. L. Olson and D. Delen, Advanced Data Mining Techniques, 1st ed. Springer Publishing Company, 2008 , p. 138.

[42] P. Viola and M. Jones, "Rapid object detection using a boosted cascade of simple features," in Proceedings of the IEEE Conference on Computer Vision and Pattern Recognition (CVPR), vol. 1, 2001, pp. 511-518.

[43] R. Lienhart and J. Maydt, "An extended set of Haar-like features for rapid object detection," in Proceedings of the International Conference on Image Processing, vol. 1, 2002, pp. 900-903.

[44] P. N. Belhumeur, J. P. Hespanha, and D. J. Kriegman, "Eigenfaces vs. Fisherfaces: Recognition Using Class Specific Linear Projection," IEEE Transactions on Pattern Analysis and Machine Intelligence, vol. 19, no. 7, pp. 711-720, July 1997.

[45] D. Chaudhuri, N. K. Kushwaha, A. Samal, and R. C. Agarwal, "Automatic Building Detection From High-Resolution Satellite Images Based on Morphology and Internal Gray Variance," IEEE Journal of Selected Topics in Applied Earth Observations and Remote Sensing, vol. 9, no. 5, pp. 1767-1779, May 2016.

[46] J. Liu, T. Fang, and D. Li, "Shadow Detection in Remotely Sensed Images Based on Self-Adaptive Feature Selection," IEEE Transactions on Geoscience and Remote Sensing, vol. 49, no. 12, pp. 5092-5103, Dec 2011. 


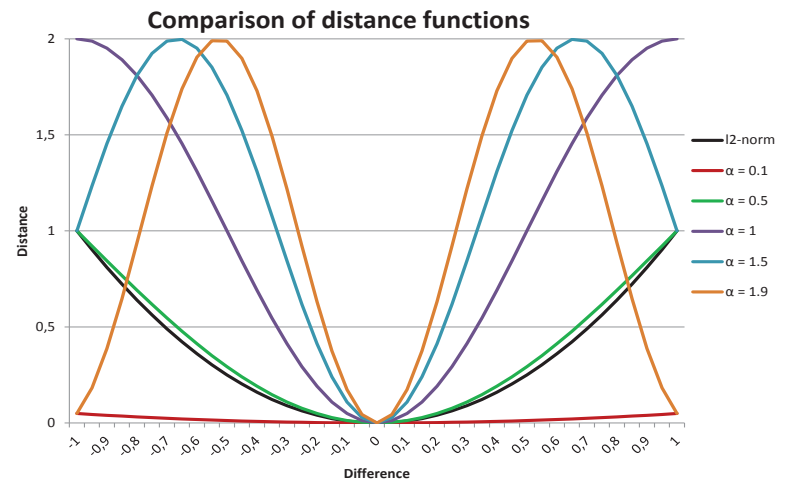

Fig. 2. Comparison of the $l_{2}$-norm and the cosine-based distance function for various values of parameter $\alpha$.

TABLE I

COMPUTATION OF DISTANCES BETWEEN FIRST ROW HISTOGRAM AND SECOND AND THIRD ROW HISTOGRAMS USING THE $l_{2}$-NORM AND THE COSINE-BASED DISTANCE FUNCTION FOR VARIOUS VALUES OF $\alpha$.

\begin{tabular}{|c|c|c|c|c|c|c|}
\hline \multirow{2}{*}{ Histograms } & \multirow{2}{*}{$\boldsymbol{l}_{2}$-norm } & \multicolumn{5}{|c|}{ Cosine-based dissimilarity measure } \\
\cline { 4 - 8 } & & $\alpha=0.1$ & $\alpha=0.5$ & $\alpha=1$ & $\alpha=1.5$ & $\alpha=1.9$ \\
\hline 1 & - & - & - & - & - & - \\
\hline 0 & 0.64 & 0.0314 & 0.691 & 1.809 & 1.809 & 0.9372 \\
\hline 0 & 0.64 & 0.0315 & 0.769 & 2.8417 & 5.5997 & 7.7742 \\
\hline
\end{tabular}

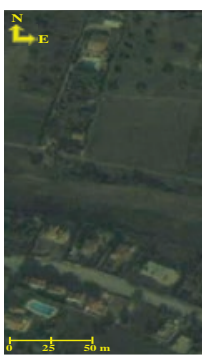

(a)

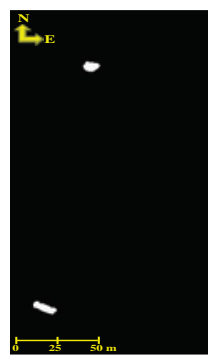

(b)

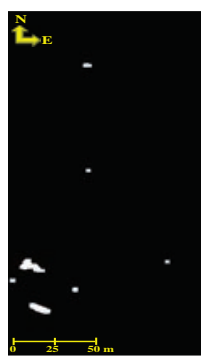

(c)

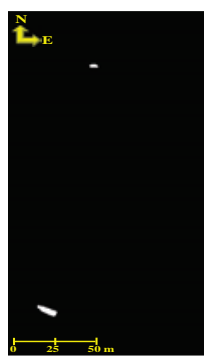

(d)

Fig. 3. Comparison of water detection methods in a problematic image of our dataset. (a) Original image. (b) Ground truth of water locations. (c) Water mask produced by $N D W I$. (d) Water mask produced by our proposed index (8). 


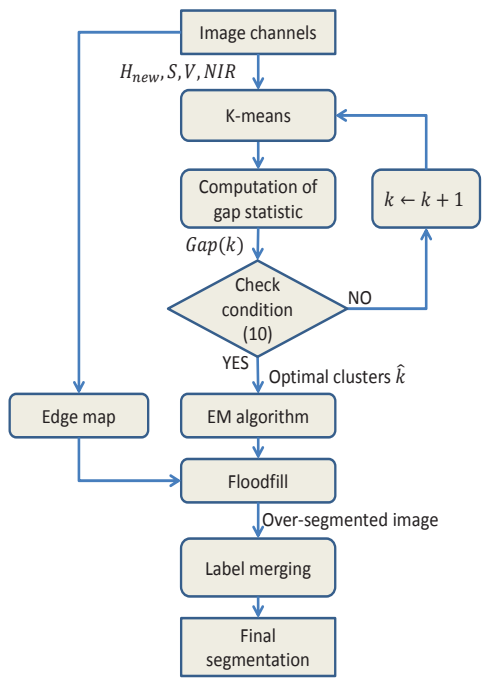

Fig. 4. Block diagram of the image segmentation procedure.

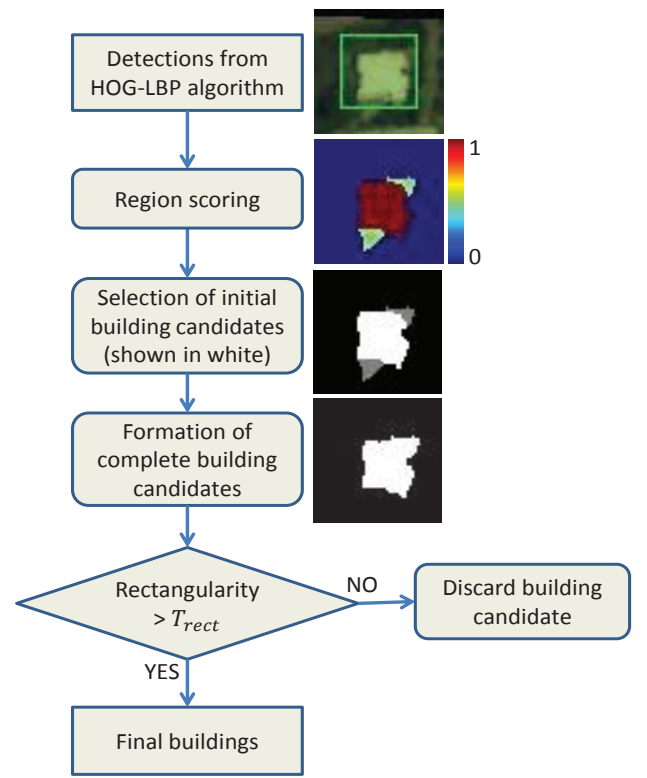

Fig. 5. Block diagram of the building region detection algorithm, along with corresponding results of running the algorithm on a HOG-LBP detection. 


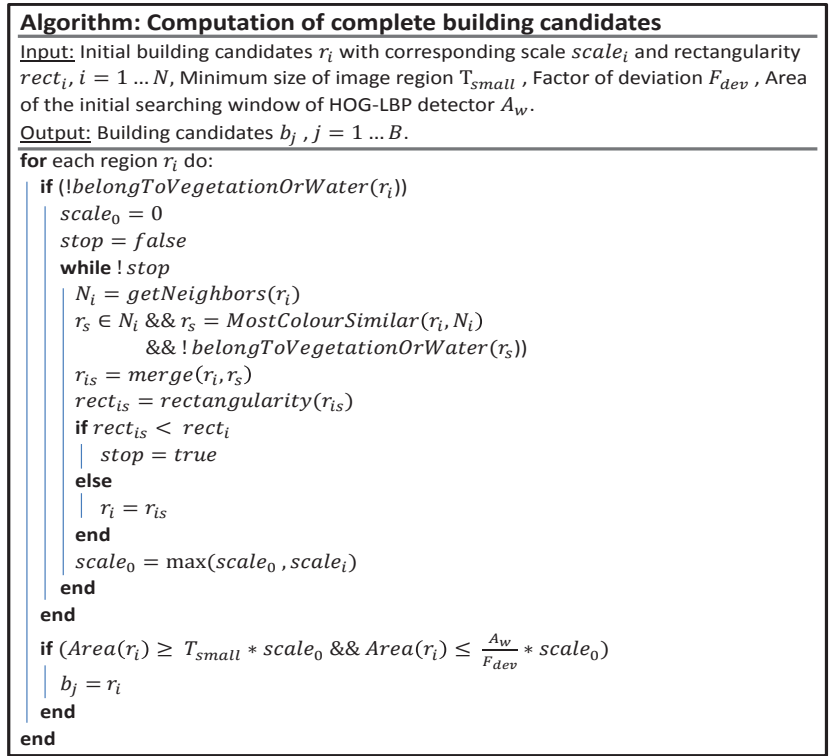

Fig. 6. Outline of the algorithm that forms the complete building candidates written in pseudocode.

TABLE II

TOTAL RECALL, PRECISION AND $F_{1}$-SCORE OF THE TESTED METHODOLOGIES AT OBJECT AND PIXEL LEVEL IN THE ATHENS DATASET. BEST VALUES SHOWN IN BOLD.

\begin{tabular}{|l|c|c|c|c|c|c|}
\hline \multirow{2}{*}{ Method } & \multicolumn{2}{|c|}{ Object-based results } & \multicolumn{3}{c|}{ Pixel-based results } \\
\cline { 2 - 8 } & Recall & Precision & F1-score & Recall & Precision & F1-score \\
\hline M1. HOG-LBP detector & 0.978 & 0.645 & 0.777 & 0.964 & 0.108 & 0.194 \\
\hline M2. HOG-LBP detector + Platt scaling & 0.951 & 0.746 & 0.836 & 0.945 & 0.121 & 0.215 \\
\hline M3. Enhanced HOG-LBP detector & 0.964 & 0.745 & 0.84 & 0.94 & 0.112 & 0.2 \\
\hline M4. Enhanced HOG-LBP detector + Platt scaling & 0.957 & 0.806 & 0.875 & 0.946 & 0.12 & 0.212 \\
\hline M5. Cascade classifier DAB with HAAR features (16 stages) & 0.764 & 0.637 & 0.695 & 0.736 & 0.21 & 0.327 \\
\hline M6. Cascade classifier LB with LBP features (17 stages) & 0.9 & 0.704 & 0.79 & 0.876 & 0.13 & 0.227 \\
\hline M7. Fischerface detector & 0.999 & 0.451 & 0.621 & 0.998 & 0.089 & 0.163 \\
\hline M8. Sirmacek et al. (2011) & 0.55 & 0.466 & 0.504 & - & - & - \\
\hline M9. Konstantinidis et al. (2015) & 0.854 & 0.605 & 0.708 & 0.825 & 0.176 & 0.29 \\
\hline M10. Chaudhuri et al. (2015) & 0.499 & 0.892 & 0.64 & 0.354 & 0.271 & 0.307 \\
\hline $\begin{array}{l}\text { M11. Enhanced HOG-LBP detector + Platt scaling + Region } \\
\text { Refinement }\end{array}$ & 0.719 & 0.724 & 0.722 & 0.555 & 0.488 & 0.519 \\
\hline
\end{tabular}




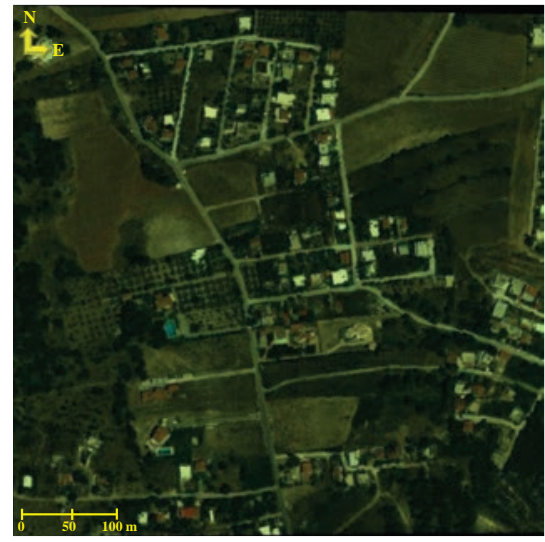

(a)

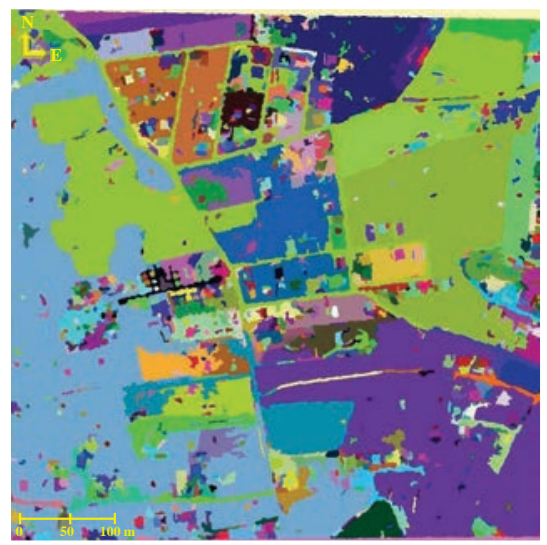

(d)

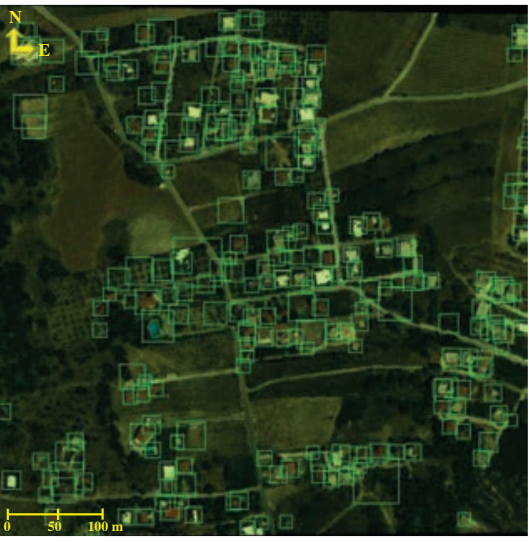

(b)

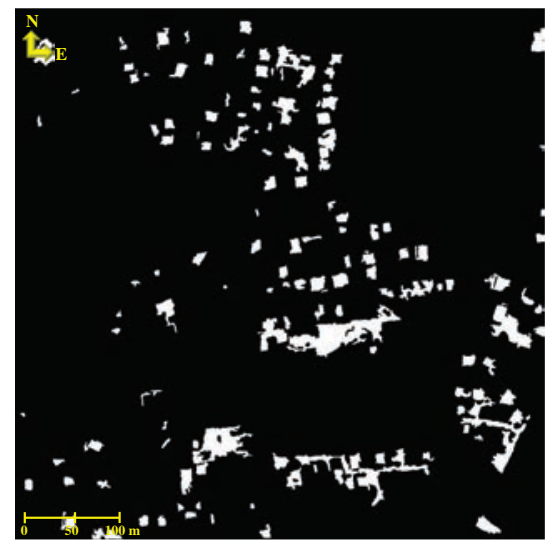

(e)

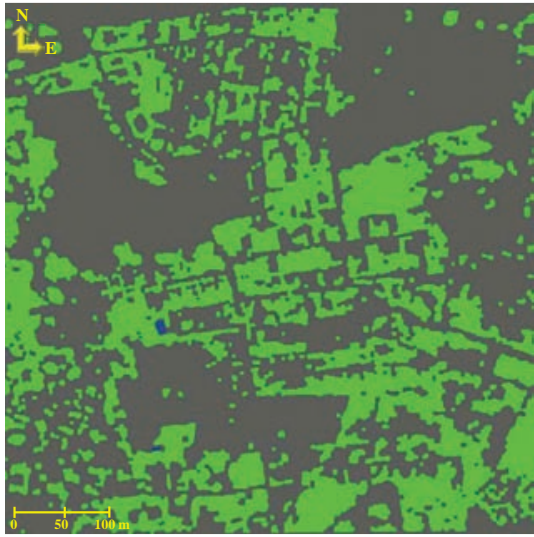

(c)

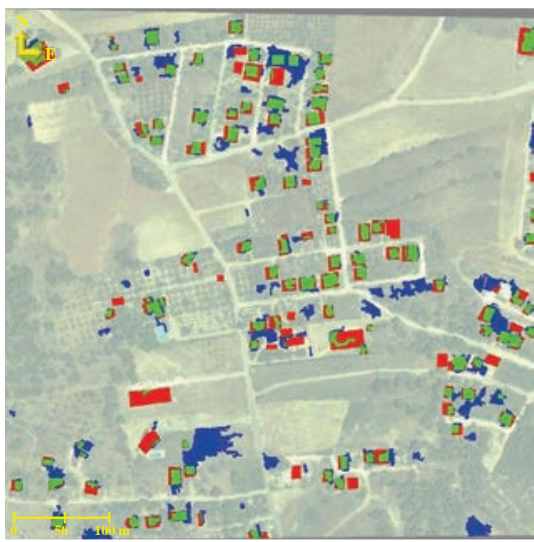

(f)

Fig. 7. Presentation of the intermediate and final results of our proposed methodology. (a) Original satellite image. (b) Detections of HOG-LBP algorithm shown as green rectangles. (c) Computed vegetation (green) and water (blue) areas. (d) Final image segmentation after region merging. (e) Initial building candidates shown in white. (f) Final buildings extracted by our method. True positives (green), false positives (blue) and false negatives (red) are depicted.

TABLE III

OBJECT-BASED RESULTS FOR COMBINATIONS OF HOG AND LBP VARIANTS. BEST VALUES SHOWN IN BOLD.

\begin{tabular}{|l|c|c|c|}
\hline \multirow{2}{*}{$\begin{array}{l}\text { Combinations of HOG and } \\
\text { LBP feature vectors }\end{array}$} & \multicolumn{3}{|c|}{ Object-based results } \\
\cline { 2 - 4 } & Recall & Precision & F1-score \\
\hline HOG + Classical LBP & 0.86 & 0.61 & 0.714 \\
\hline HOG + Uniform LBP & 0.866 & 0.603 & 0.711 \\
\hline HOG + CS-LBP & 0.864 & 0.495 & 0.629 \\
\hline HOG + ID-LBP & 0.848 & 0.596 & 0.7 \\
\hline
\end{tabular}




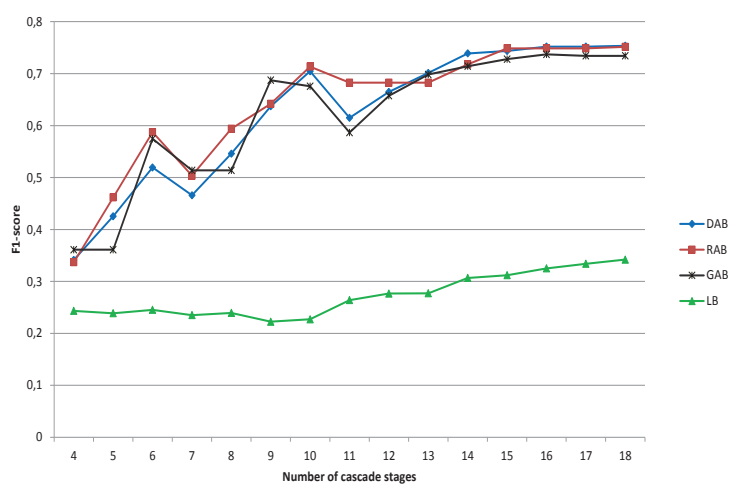

(a)

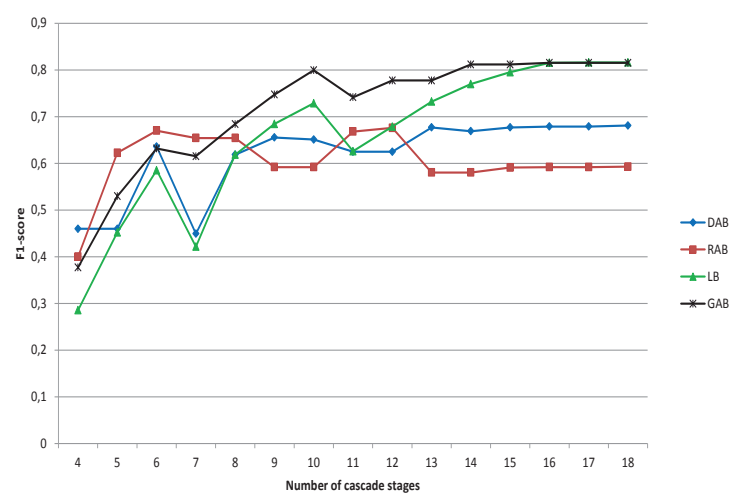

(b)

Fig. 8. Presentation of the achieved $F_{1}$-score for the tested configurations of cascade adaboost classifiers on the test set. (a) Cascade HAAR classifier. (b) Cascade LBP classifier. DAB: Discrete Adaboost, RAB: Real Adaboost, LB: Logitboost, GAB: Gentle Adaboost

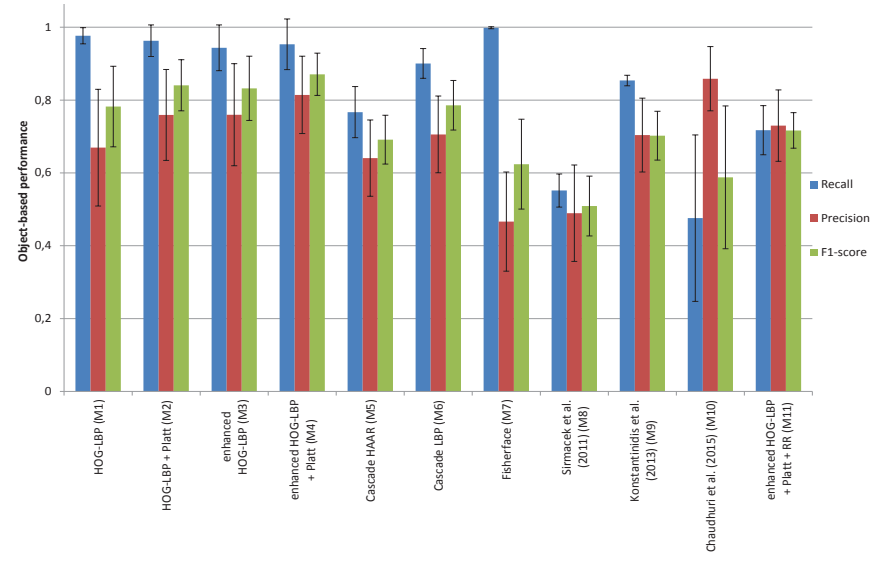

Fig. 9. Average values of recall, precision and $F_{1}$-score and their standard deviations for the tested methodologies at object level in the Athens dataset. RR: Region Refinement. 


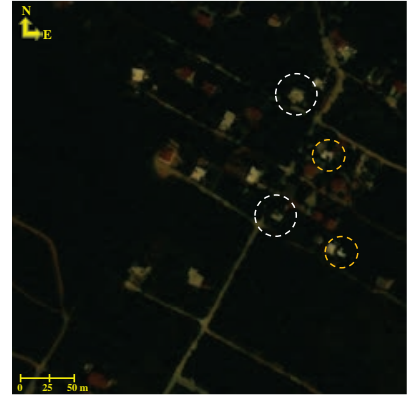

(a)

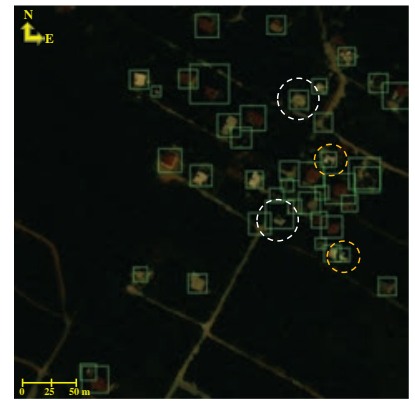

(e)

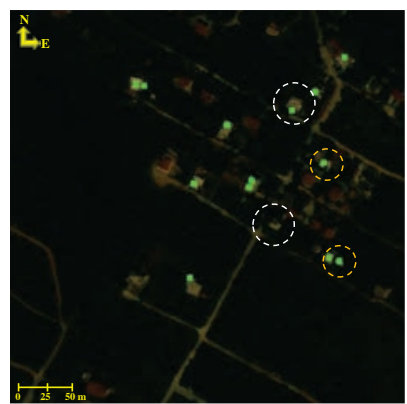

(i)

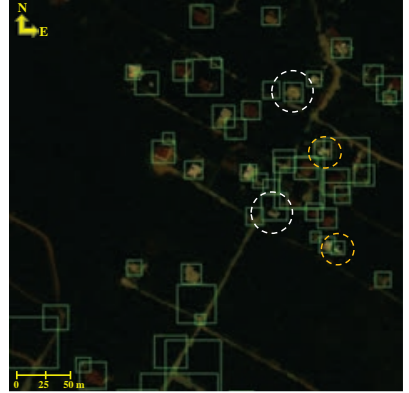

(b)

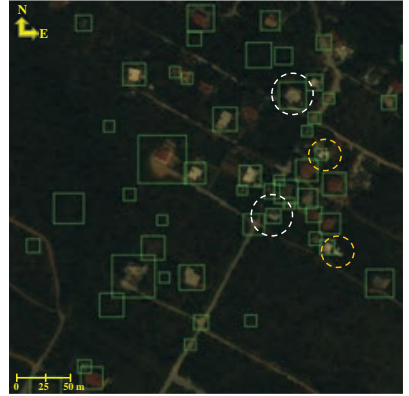

(f)

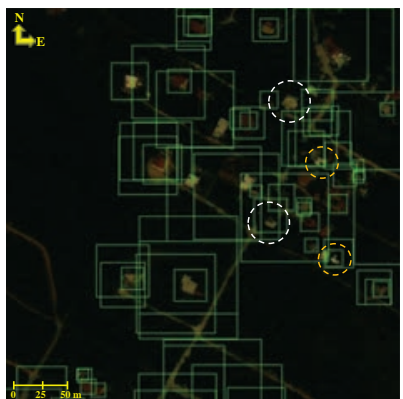

(j)

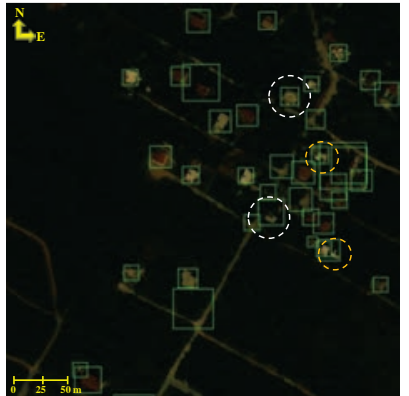

(c)

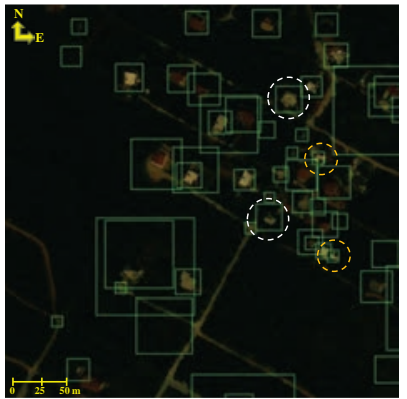

(g)

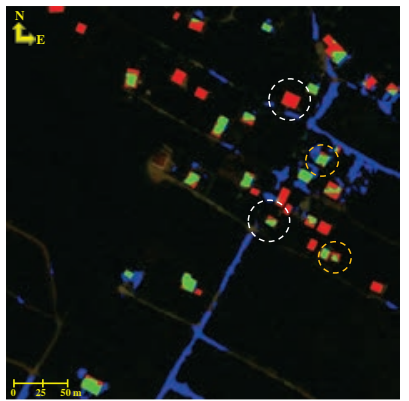

(k)

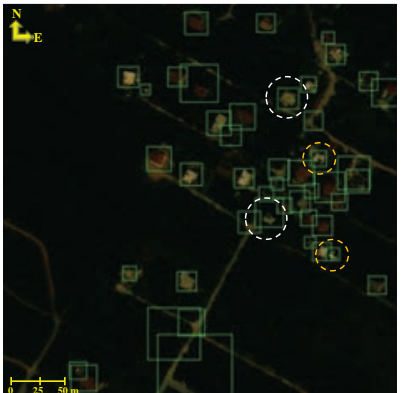

(d)

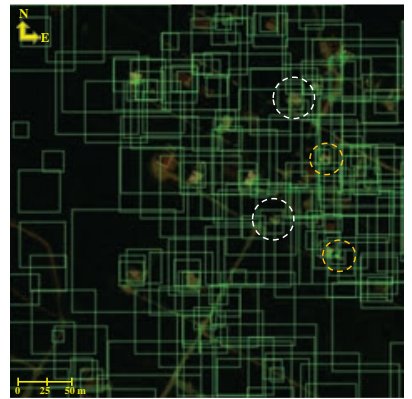

(h)

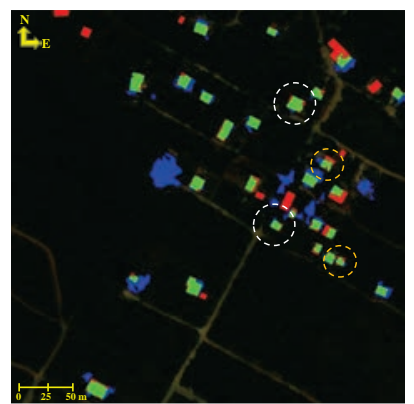

(1)

Fig. 10. Comparison of the tested methodologies in a part of a satellite image depicting buildings with spectral variations in their rooftops (white circles) and buildings covered partially by vegetation (orange circles). The initial image is presented in (a), while the corresponding results of algorithms M1-M11 are shown in (b)-(1).

TABLE IV

PARAMETER SETTINGS FOR THE PROPOSED APPROACH.

\begin{tabular}{|c|c|}
\hline Parameter & Value \\
\hline Frequency of cosine-based measure $(\alpha)$ & 0.09 \\
\hline Subsets of data for gap statistic $(B)$ & 20 \\
\hline Minimum image region $\left(T_{\text {small }}\right)$ & 30 pixels \\
\hline Factor of deviation $\left(F_{\text {dev }}\right)$ & 0.45 \\
\hline Overlap threshold with vegetation or water $\left(T_{\text {over }}\right)$ & $30 \%$ \\
\hline Weight vector $\boldsymbol{w}$ & {$[0.4,0.1,0.8,0.1]$} \\
\hline Rectangularity threshold $\left(T_{\text {rect }}\right)$ & 0.4 \\
\hline
\end{tabular}




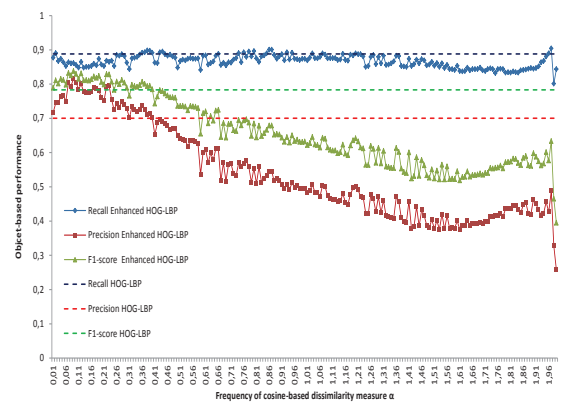

(a)

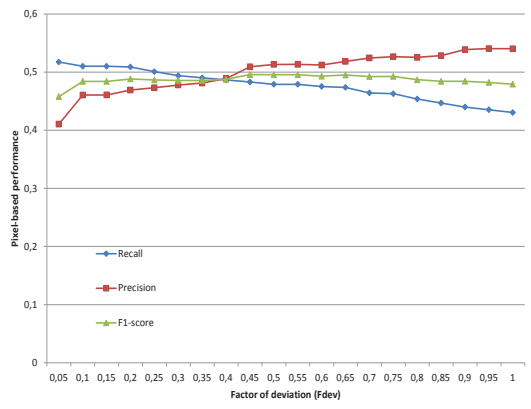

(d)

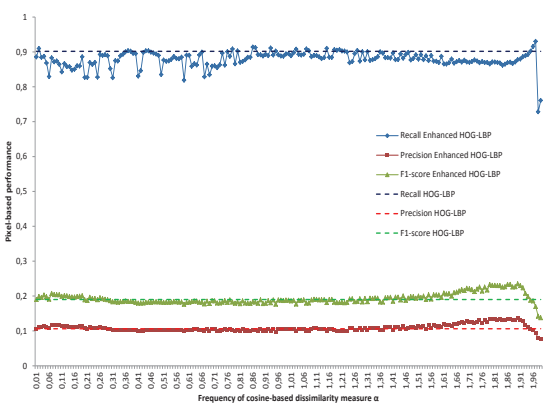

(b)

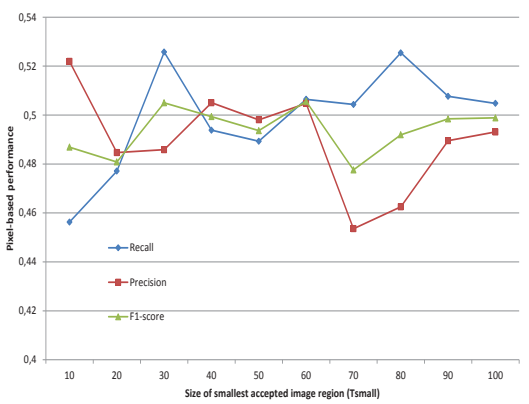

(e)

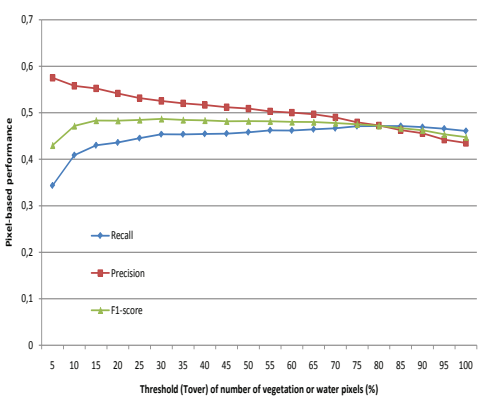

(c)

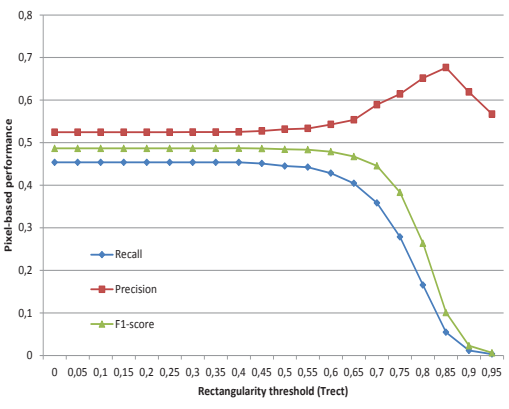

(f)

Fig. 11. Performance of our proposed methodology on the validation set for different parameter settings. (a) Effect of frequency $\alpha$ on object-based performance. (b) Effect of frequency $\alpha$ on pixel-based performance. (c) Effect of overlap threshold with vegetation and water $T_{\text {over }}$, (d) effect of factor of deviation $F_{d e v}$, (e) effect of size of smallest accepted region $T_{s m a l l}$ and (f) effect of rectangularity threshold $T_{\text {rect }}$ on pixel-based performance. 


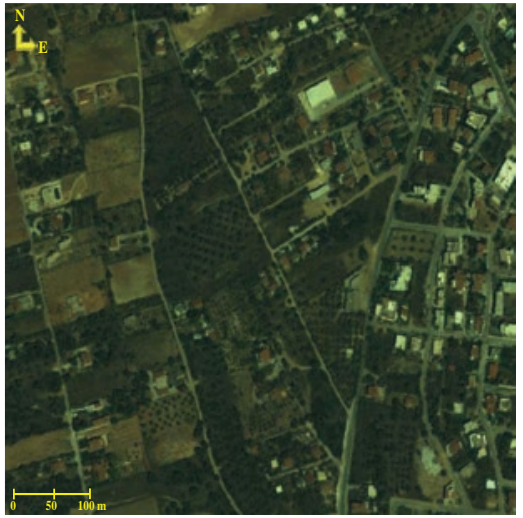

(a)

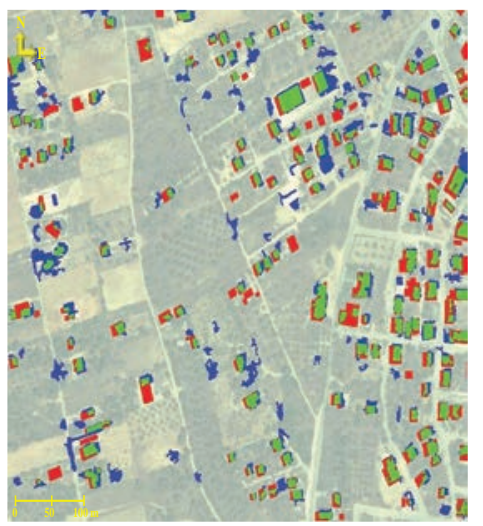

(d)

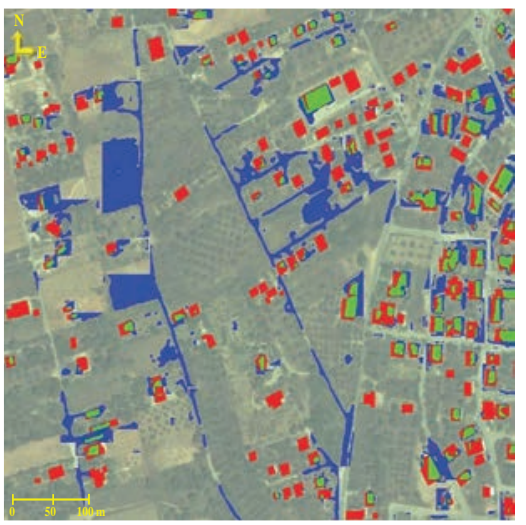

(g)

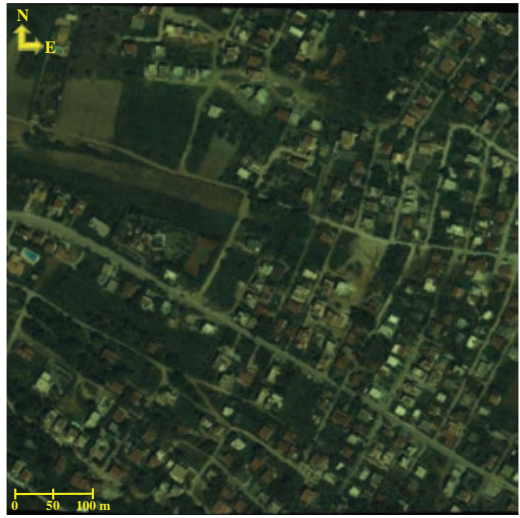

(b)

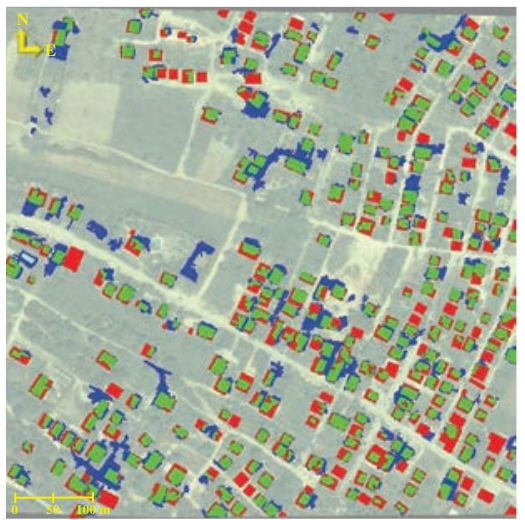

(e)

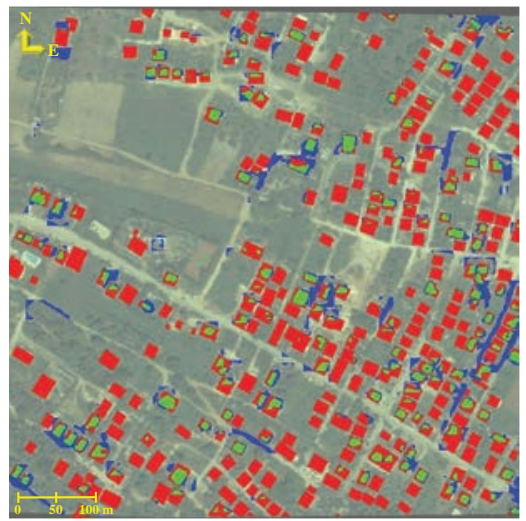

(h)

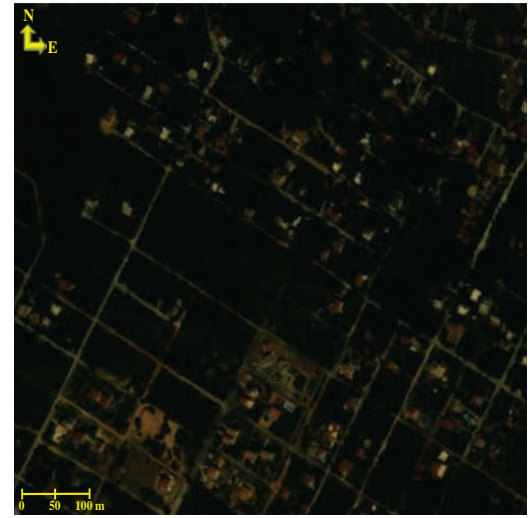

(c)

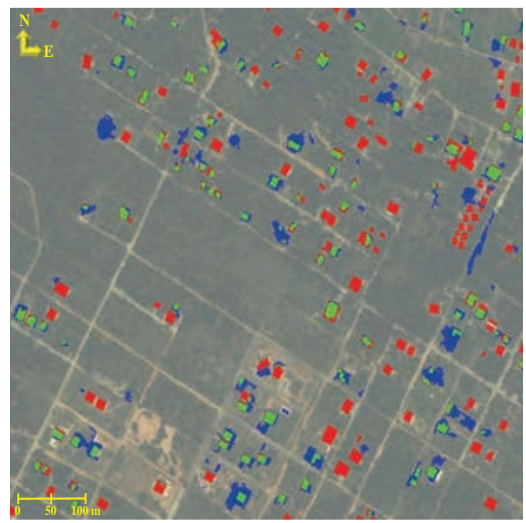

(f)

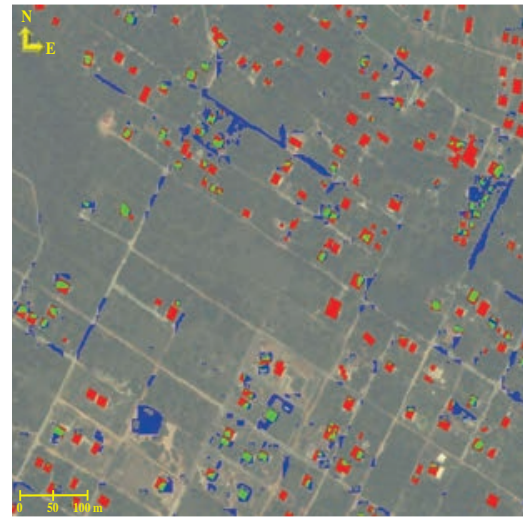

(i)

Fig. 12. Comparison of our proposed methodology and the method proposed by Chaudhuri et al. [45] in 3 images of the Athens dataset. (a),(b) Original QuickBird images. (c) Original WorldView-2 image. (d)-(f) Detected buildings from our method (M11) for images (a)-(c) respectively. (g)-(i) Detected buildings from the method of Chaudhuri et al. (M10) for images (a)-(c). True positive pixels are shown in green, false positive pixels are shown in blue and missed true positive pixels are presented in red. 